\title{
Radiation Transport
}

\section{July 1, 1985-September 30, 1985}

\author{
R. D. O'Dell
}

DISCLAIMER

\begin{abstract}
This report was prepared as an account of work sponsored by an agency of the United States Government. Neither the United States Government noi any agrincy thereof, nor any of their employees, makes any warranty, express or implied, or assumes any legal liability or responsibility for the accuracy, completeness, or usefulness of any information, apparatus, product, or process disclosed, or represents that its use would not infringe privately owned rights. Reference herein to any specific commercial product, process, or service by trade name, trademark, manufacturer, or otnerwisc does not necessarily constitut : or imply its endorsement, recommendation, or favoring by the United States Government or any agency thereof. The views and opinions of authors expressed herein do not necessarily state or reflect those of the United States Government or any agency thereof.
\end{abstract}


56. R. C. Little and R. E. Seamon, "Continuous-Energy Cross Sections for Eu, $\mathrm{Gd}$, and Ho-165 from ENDL85," Los Alamos National Laboratory memorandum $X-6:$ RCL : RES $-85-453$ (September 11, 1985).

57. R. E. Seamon, "ENDL-85 Multigroup Cross Sections for Eu, Gd, and Ho-165," Los Alamos National Laboratory memorandum X-6:RES-85-461 (September 10, 1985).

58. R. E. Seamon and R. C. Little, "Multigroup Cross Sections for Eu, Gd, and Ho-165 from ENDL85," Los Alamos National Laboratory meminandum $X-6:$ RES : RCL $-85-468$ (September 20, 1985).

59. J. A. Halblelb, Sr., and W. H. Vandevender, "CYLTRAN: A Cylindrical Geometry Multimaterial Electron/Photon Monte Carlo Transport Code," Sandia National Laboratories report SAND 74-0030 (March 1975).

60. H. M. Colbert, "SANDYL: A Computer Code for Calculating Combined PhotonElectron Transport in Complex Systems," Sandia National Laboratories report SLL-74-0012 (Livermore, California, 1973).

61. M. J. Eerger and S. M. Seltzer, "Electron and Photon Transport Programs," Part I, "Introduction and Notes on Program DATAPAC 4," National Bureau of Standards report NBS-9836 (U.S. Department of Commerce, Washington, D.C., June 1968).

62. M. J. Berger and S. M. Seltzer, "ETRAN Monte Carlo Code System for Electron and Photon Transport Through Extended Media," Oak Ridge National Laboratory report CCC-107 (1968). 


\section{CONTENTS}

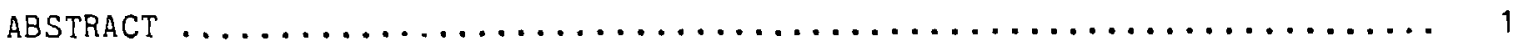

I. INTRODUCTION $\ldots \ldots \ldots \ldots \ldots \ldots \ldots \ldots \ldots \ldots \ldots \ldots \ldots \ldots \ldots \ldots \ldots \ldots$

II. DETERMINISTIC RADIATION TRANSPORT $\ldots \ldots \ldots \ldots \ldots \ldots \ldots \ldots \ldots \ldots \ldots$

A. A New Application for ONEDANT (R. D. O'Dell and

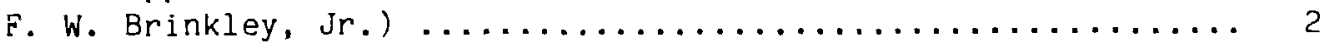

B. ONEDANT/TWODANT Maintenance and Support (F. W. Brinkley, Jr.) . 6

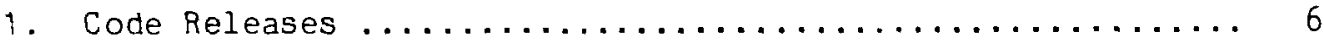

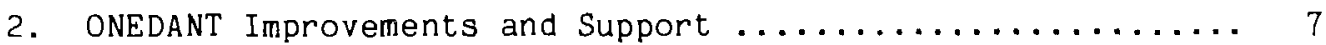

3. TWODANT Maintenance and Support ................. 7

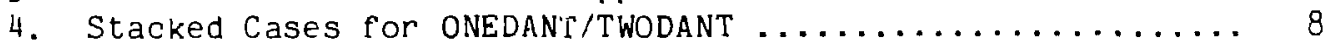

C. Th'DHEX Code Support (F. W. Brinkley, Jr.) $\ldots \ldots \ldots \ldots \ldots \ldots \ldots$

D. TWODANT Improvements (R. E. Alcouffe) ............... 9

E. Two-Dimensional Radiation Transport (R. E. Alcouffe) ....... 9

F. Asymptotic Solutions of Numerical Transport Problems in

Diffusive Regimes ( $E$. $W$. Larsen and W. F. Miller, Jr.) ...... 23

G. Maximal Principles for Radlative Transfer Problems

(E. W. Larsen) ........................... 27

H. Linking Monte Carlo and $S_{N}$ via Response Matrices

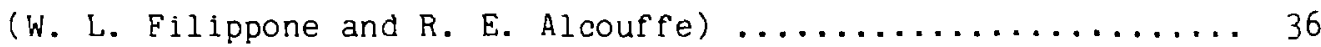

1. Precalculated Response Matrices ................ 38

2. Accelerating the Interface Flux Iterations $\ldots \ldots \ldots \ldots \ldots \ldots 41$

3. Sample Calculations ..................... 45

II. MONTE CARLO RAdIATION TRANSPURT $\ldots \ldots \ldots \ldots \ldots \ldots \ldots \ldots \ldots \ldots \ldots$

A. MCNP Support (J. Briesmeister) $\ldots \ldots \ldots \ldots \ldots \ldots \ldots \ldots \ldots \ldots \ldots$

B. Biasing Through a Cylindrical Window from a Sphericaliy

Symmetric Surface Source in MCNP3A (E. C. Snow) .......... 47

C. Elimination of the Law iô Sampling Scheme from MCNF
(H. M. Eisher) $\ldots \ldots \ldots \ldots \ldots \ldots \ldots \ldots \ldots \ldots \ldots \ldots \ldots \ldots \ldots \ldots \ldots \ldots \ldots \ldots \ldots \ldots \ldots$

D. HETC and Related Improvements (R. E. Prael) ............ 65

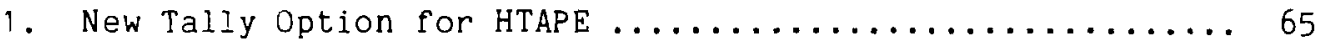

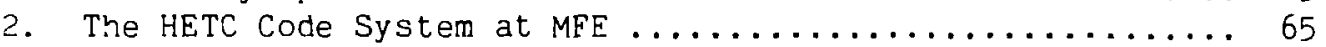

3. Antiproton Annihilation Source for HETC .............. 65

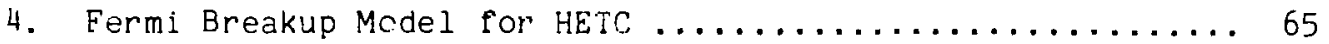

E. Ring Tally for CYLTRAN ( $H$. G. Hugines) $\ldots \ldots \ldots \ldots \ldots \ldots \ldots \ldots 66$

IV. CROSS SECTIONS AND PHYSICS $\ldots \ldots \ldots \ldots \ldots \ldots \ldots \ldots \ldots \ldots \ldots$

A. Cross-Section Processirig Codes on the CRAY (R. C. Little) .... 69

B. Fission Produci Cross Sections (R. E. Seamon and R. C. Little). 70

C. Multigroup Charged Particle Data (R. C. Little and

R. E. Seamon) ............................. 71 
D. New Data (R. C. Little and R. E. Seamon) .............. 72

1. Low-Energy Photon Cross Sections for MCNP .............. 72

2. Sc -45 Cross Sections for MCNP .................... 73

3. $Y-89$ Cross Sections for MCNP ................. 73

4. Cross Sections for Eu, Gd, and Ho-165 from ENDL-85 in Continuous-knergy and Multigroup Form .............. 74

E. Physics Sources of the ITS Coupled Electron/Photon Monte Carlo Transport Codes (Particularly CYLTRAN) (J. J. Devaney) ....... 75

REFERENCES $\ldots \ldots \ldots \ldots \ldots \ldots \ldots \ldots \ldots \ldots \ldots \ldots \ldots \ldots \ldots \ldots \ldots \ldots \ldots \ldots \ldots \ldots$ 
RADIATION TRANSPORT

July 1, 1985 - September 30, 1985

by

R. D. O'Dell

\section{ABSTRACT}

Research and development progress in radiation transport by the Los Alamos National Laboratory's Group $X-6$ for the fourth quarte: of FY 85 is reported. Included are unclassifled tasks in the areas of Deterministic Padiation Transport, Monte Carlo Radiation Transport, and Cross Sectior:- and Physics.

\section{INTRODUCTION}

Research, development, and design analyses performed by Group $X-6$, Radiction Transport, of the Applied Theoretical Physics Division during the fourth quarter of FY 85 are described in this progress report. Included is the unclassified portion of programs funded by the US Department of Energy. Our classified work is reported elsewhere. Some of the reported work was performed in direct support of other Laboratory groups and other contractors.

This report 19 divided into three sections: Deterministic Radiation Transport, Monte Carlo Radiation Transport, and Cross Sections and Physics. Authors of individual task reports are listed in parentheses after each task title. Authors not in Group $x-6$ also have their affiliation noted. Readers are ensouraged to contact these cognizant personnel directly for additional information or further published resulto.

Technical program management for Group $X-6$ during this reporting period was provided by R. Arthur Forster, Group Leader, and by Associate Group Leaders John S. Hendricks, R. Douglas O'Dell, and Billy D. Meixner. 


\section{DETERMINISTIC RADIATION TRANSPORT}

The deterministic radiation transport effort in Group $\mathrm{X}-6$ is involved in the development and assessment of both numerical and analytical methods and models ror deterministic radiation transport; in the development and testing of new and improved deterministic transport codes; in the maintenance, improvement, and support of existing computer codes; and in the application of our codes to nuclear analyses. In this report we describe some of the work we have been doing in our deterministic transport effort.

A. A New Application for ONEDANT (R. D. O'Dell and F. W. Brinkley, Jr.)

During this reporting period we were contacted by $W$. C. Feldman of Group ESS-8 regarding an interesting computational problem. Given a space-energy cosmic ray-induced neutron source distribution in the atmosphere of Mars, is it possible to detect the presence of varying amounts of water in the soil from neutron measurements in the upper regions of the atmosphere? If so, does $X-6$ have any computer codes that could be used for such calculations? As we discussed the problem, it Decame evident that our one-dimensional discreteordinates code ONEDANT ${ }^{1}$ could be used in the plane geometry option to calculate the multigroup energy spectrum of the neutron partial current (outflow) exiting the atmosphere. The question then remained whether or not the energy spectrum would be sensitive to the presence and amount of water (ice) in the soil of Mars.

Because of our familiarity with ONEDANT and discrete-ordinates calculations, alon; with a rather pressing time frame imposed on ESS-8, we agreed to set up the problem, run a few typical cases, perform some parametric calculations on the effects of mesh/quadrature, and provide some edit/graphics output capabilities for subsequent use by ESS-8. This report describes sorne of our work on this problem.

A rudimentary model problem was set up, as shown in Fig. 1. The atmosphere, having an areal density of $15 \mathrm{~g} / \mathrm{cm}^{2}$ at the planet's surface, was composed of carbon dioxide and nitrogen in amounts specified by Darrell crake of ESS-8. The soil was modeled in two distinct layers: a surface layer, $10 \mathrm{~g} / \mathrm{cm}^{2}$ thick, of pure, dry soil and a second layer, $120 \mathrm{~g} / \mathrm{cm}^{2}$ thlck, of soll and small amounts of water. The choice of $10 \mathrm{~g} / \mathrm{cm}^{2}$ as the thickness of the surface layer of water-free soll was arbitrary. The layering was chosen to approximate the likelihood that any water in the form of ice probably exists 


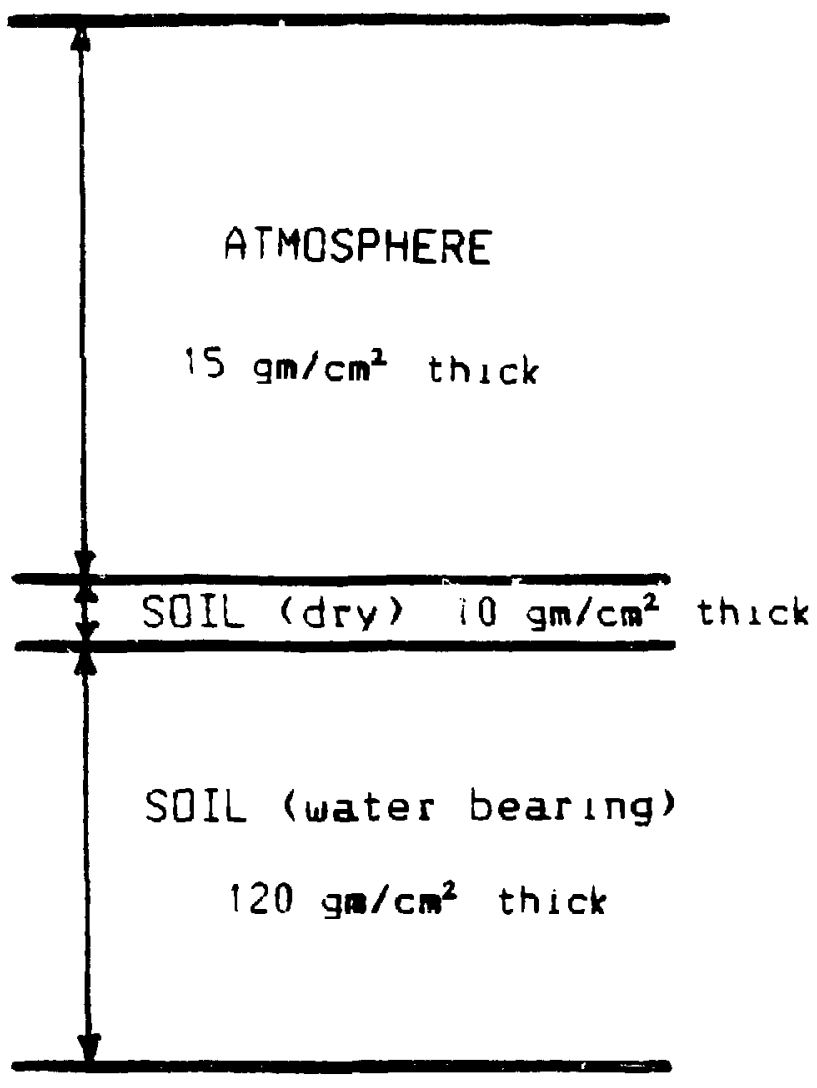

Fig. 1. Model problem of Mars for ONEDANT. 
only below the surface since surface water would tend to sublime. The soil was composed of oxides of silicon, aluminum, iron, magnesium, calcium, and other elements in quantities specified by ESS-8. For the initial calculations all densities, $\rho$, were set to $1 \mathrm{~g} / \mathrm{cm}^{3}$. The space-energy distribution of the cosmic ray-induced isotropic neutron source was provided. For our studies a 69-group (49 fast groups, 20 thermal groups) cross-section set provided by Group T-2 was used.

Our first set of calculations was scoping studies to determine the "best" values of mesh spacing, quadrature order, and thickness of the water-bearing soil. A mesh spacing, $\rho \Delta x$, of $1 \mathrm{~g} / \mathrm{cm}^{2}$ was found to be quite adequate. An $S_{8}$ Galiss-Legendre quadrature was selected because it yielded results that were within $1 \%$ of the $S_{\infty}$ results. With a water-bearing soil depth of $120 \mathrm{~g} / \mathrm{cm}^{2}$, the upper-atmosphere exiting partial currents were very nearly the values cbtained with greater thicknesses. In other words, a water-bearing soll depth of $120 \mathrm{gm} / \mathrm{cm}^{2}$ is effectively an infinite-medium depth for computational purposes.

The quantity of interest that we chose was the partial current ( $\mathrm{J}^{\text {out }}$ ) of neutrons exiting the atmosphere since this is what a $2 \pi$ detector facing the surface of the planet would see. This partial current for energy group $g$ is calculated simply as

$$
\mathrm{J}_{\mathrm{g}}^{\text {out }}=\sum_{\text {out }} \mu_{\mathrm{m}} \psi_{\mathrm{m}, \mathrm{g}^{\mathrm{w}}} \text {, }
$$

where $m$ denotes a discrete direction of neutron motion; $\mu_{m}$ is the cosine of the angle between the normal to the planet's surface and the direction (m) of neutron motion; $\psi_{m, g}$ is the angular flux of neutrons for energy group $g$ and direction $m$; and $w_{m}$ is the quadrature weight associated with $\mu_{m}$. Tre summation in Eq. (1) is made only over outgoing (exiting) directions.

The ONEDANT code was modified to print a file containing the values $\mathrm{J}_{\mathrm{g}}^{\text {out }}$. A separate code was written to produce a plot file of $\mathrm{J}_{g}^{\text {out }} / \Delta E_{g}$, where $\Delta E_{g}$ is the energy width of group $g$. With such a plot file the energy spectrum of $J_{g}$ can be plotted and comparisons can be made between the spectra as a function of water content in the underlying soll of the planet.

The difference in the energy spectrum of $\mathrm{J}^{\text {out }}$ with varying amounts of water in the soil was found to be quite pronounced. Figure 2 shows a plot of the spectrum with no water in the soil compared with that with 1\% (by welght) 


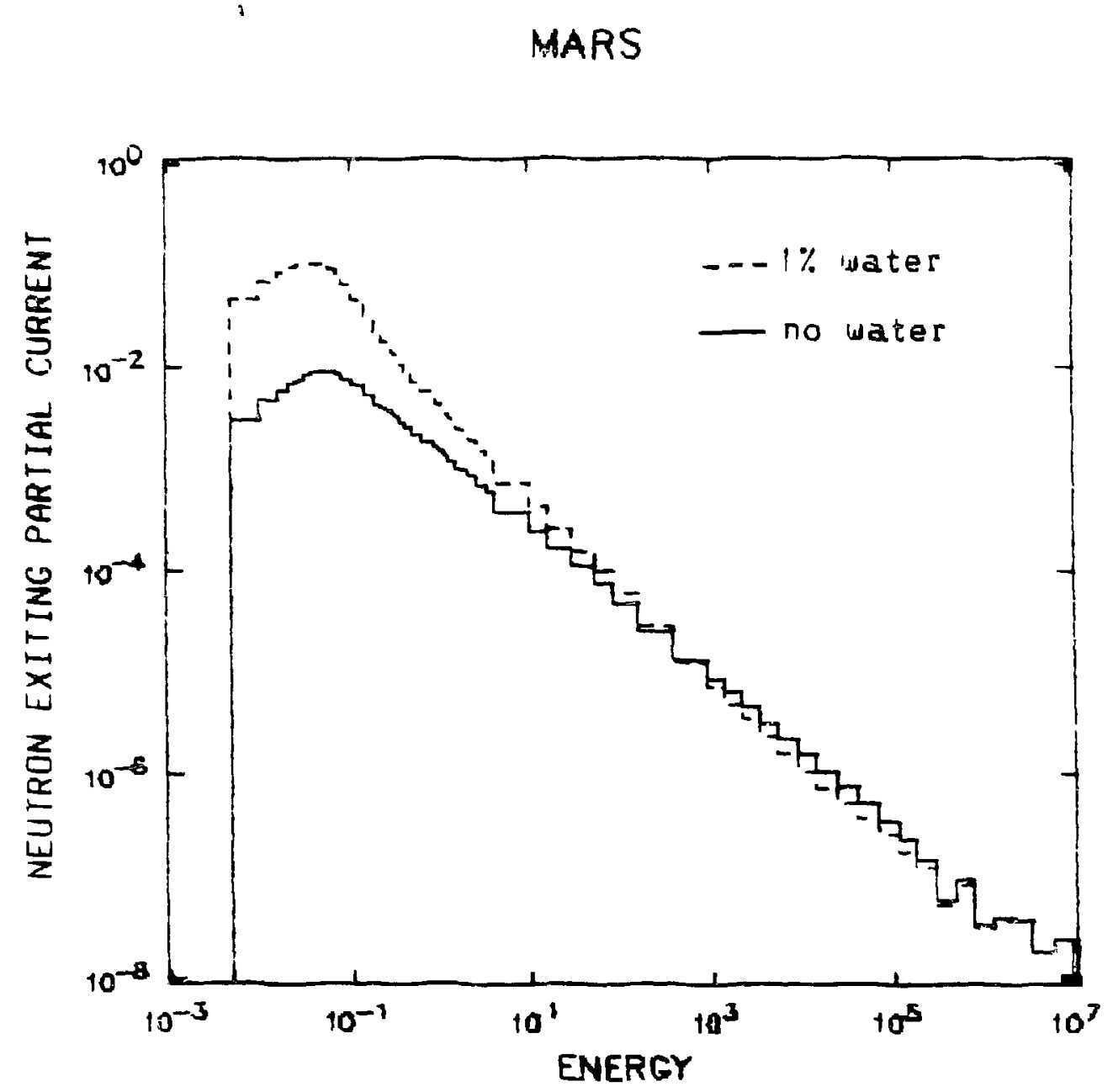

Fig. 2. Partial current spectrum exiting the atmosphere of Mars model problem. 
water. Note that the spectrum below about $0.1 \mathrm{eV}$ with only $1 \%$ water is enhanced by over an order of magnitude (factor of 12) from that with no water. Even with only $0.1 \%$ water in the soil the thermal portion of the spectrum is a factor of two larger than that with no water present.

As a note of interest, a typical ONEDANT calculation $\left[145\right.$ mesh points, $S_{8}$ quadrature, 69 groups ( $49 \mathrm{fast}, 20$ thermal), converged to $10^{-5} \mathrm{~J}$ requires about 15 sec of CPU time on our CRAY-1 computer.

Because reutrons have a finite mean lifetime, $\tau$, of about $900 \mathrm{sec}$, there is finite probability that neutrons $w_{\perp} l l$ be lost by decay before they escape or are absorbed in the atmosphere of Mars. The characteristic "decay Iength" for neutrons is $\tau \nu_{g}$, where $\nu_{g}$ is the neutron velocity. The reciprocal of this decay length can be considered an effective decay cross section. Since this effective decay cross section is largest for low-energy neutrons where $v_{g}$ is smallest, a question arose about the sensitivity of low energy exiting partial current to neutron decay. To account for this, ONEDANT was modifled so the effective decay cross section $\left(\tau \nu_{g}\right)^{-1}$ could be added to the total cross section as a user option. The option was made available for fixed source, source plus fission, and $k_{\text {eff }}$ problems (IEVT<2) and is flagged by setting IPVT=3 with the value of $T$ entered as $P V$ in the solver module input. Effects of including neutron decay will be reported in the next quarter.

B. ONEDANT/TWOOANT Maintenance and Support (F. W. Brinkley, Jr.)

Our discrete-ordinates code packages ONEDANT ${ }^{1}$ and TWODANT $^{2}$ continue to be used steadily within and sutside the laboratory. We now describe some of the work performed in support of these codes.

1. Code Releases. The Cray Operating System (COS) versions of ONEDANT and TWODANT were sent to both RSIC (Radiation Shielding Information Center) and NESC (National Energy Software Center) for general release and distribution. Even though the two codes share common Input and Edit Todules, the TWODANT Solver module is limited to US distribution, whereas the ONEDANT Solver module enjoys unrestricted distribution. Accordingly, ONEDANT and TWODANT were released to the code centers as separate, independent code packages.

During the reporting period, J. Philoin at Sandia National Laboratories, Albuquerque (SNLA), was able to find time to implement a previously sent ONEDANT/TWODANT COS package onto the SNLA COS system under the CFT 1.13 
compiler. As we had expected, a few minor changes were needed to mako the code operational, but these corrections were supplied and effected within the day.

A similar CoS package was sent to P. $\dot{A}$. Harris of Hockwell International in response to his request.

2. ONEDANT Improvements and Support. At the request of $S$. Gerstl of T-DOT, a special albedo boundary condition patch prepared by X-6 in 1983 was brought up to date for our current version of ONEDANT. A special version of the current ONEDANT was created and supplied to Gerstl.

In support of the ESS-8 application of ONEDANT to subsurface water detection on Mars, as reported in the preceding section of this report, a plotting package was written to display the spectrum of the exiting partial current. We chose to use the CGSHIGH graphics package to do this. The plotting package permits the plotting of multiple spectra obtained from multiple ONEDANT rung. An energy group cutoff option was included at the request of ESS-8. We also added the capability to produce color graphics with each spectrum plotted in a different color.

3. TWODANT Mairtenance and Support. Some runs made with our TWODANT code uncovered a computing system bug. 3uffers were not properly aligned, resulting in the destruction of certain buffers. C Division promptly fixed this error. We also found that the Input module was raquiring about twice as much execution time as it had previously. This occurred after a C-Division library change. We traced the source of tris problem to a character-compare routine which had been substituted in the library. When another routine was used in its place, execution times returned to normal.

Several bugs in our diagonal-sweep ${ }^{3}$ version of TWODANI were discovered and corrected with the assistance of $R$. Alcouffe of $X-6$. One of these bugs occurred in a fixed source problem contaiaing void regions. Negative flux $f i x u p s$ in the void were producing nonphysical peaks in the scalar flux. C. Simmer of $T-D O T$ reported that the code-dependent angular-flux-moments file RMFLUX generated by TWODANT did not coritain the correct high-order moments. We found that the cause of the bug was that in the diagonal-sweep version the higher-order angular-flux moments are not stored contiguous to the scalar fluxes. This was corrected. J. Sapir of $Q-12$ reported a problem in which the code refused to execute an edit-only problem in which the cross-section library contained only edit cross sections. This was corrected. C. Adams of Argonne 
National Laboratory (ANL) brought to our attention an error caused by a onegroup cross-section set which contained no self-scatter. This caused the sode to read the wrong record for the scattering-index information. This bug was corrected and the corrections were relayed to ANL.

4. Stacked Cases for ONEDANT/TWODANT. Ray Alcouffe of $x-6$ requested that we provide the capability to run stacked cases, that is, multiple problems in a single input setup in ONEDANT and TWODANT. We were able to readily accommodate his request with the restriction that binary output files--for example, the RTFLUX scalar flux standard interface file ${ }^{4}$-would not be saved when the next case was run. Also, a fatal input error in a case will terminate the entire run.

To run stacked cases we use the delimiter Jeof, beginning in column 1. Thus, the form of input for three independent cases (problems) is:

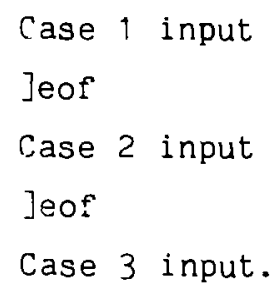

Jeof

Case 2 input

Jeof

Case 3 input.

C. TWOHEX Code Support (F. W. Brinkley, Jr.)

In using our TwOHEX triangular mesh, discrete-ordinates code package, 5 $\mathrm{J}$. Sapir of Q-12 reported a bug in the code's treatment of user-input geometric buckling. We readily corrected this bug and, at the same time, brought TWOHEX up to date using our latest versions of our standardized Input and Edit modules. Recall that TWOHEX uses the same Input and Edit modules as ONEDANT ${ }^{1}$ and TWODANT. 2

Also, C. Adams of ANL had expressed interest in receiving TWOHEX at Argonne for use in ANL's IBM computing environment. We modified our CRAY version of TWOHEX by replacing our Los Alamos "conditional vector" function calls with regular FORTRAN statements and by replacing a few Cray SCILIB routines with standard FORTRAN routines. The new IBM-compatible version was sent to ANL. 
A basic improvement for pure source problems has been added to the TWODANT code; $^{2}$ this is the source-corrected, diffusion-accelerated iteration method. ${ }^{6}$ For some geometrically complicated $p$ ublems with regions of low density or volds, it has been experimentally determined that the sourcecorrected method is more stable than other methods when many flux fixups occur. Thus, we have Implemented this method in TwODANT for pure source problems--that is, no outer iterations required--and we have tested it on some typical problems. For example, we use a geometrically complex problem, as shown in Fig. 3. In this problem we have a source centered near the origin and are asked to find the flux throughout the medium. The problem was run with 30 energy groups, $S_{8}$ with $P_{1}$ scatter, and with a $26 \times 110$ spatial mesh. When this was run in the diffusion-corrected mode, we required 390 inner iterations and 93.6 CPU sec. In the source-corrected mode, we require 329 inner iterations and 80.0 CPU sec to converge. This improvement is due to the better interaction of the source-corrected method with the fixups in selected groups. Thus, based upon this and similar experience, we have chosen the source correction method for all nonisissioning source problems.

A second improvement to the TWODANT code is the addition of a plotting package as a postprocessor. Briefly, this package takes the RTFLUX (scalar flux) file and the GEODST geometry file $e^{4}$ and plots the two-dimensional flux as a three-dimensional projection. If the fission file is present, it will also plot the fissions. Also in accordance with user instructions, one-dimensional traverses of the fluxes through the problem can be plotted. This package is available on an experimental basis from F. W. Brinkley, who is making improvements to it. This type of graphical output is very useful for assessing the quality of the calculated fluxes and for extracting needed information.

E. Two-Dimensional Radiation Transport (R. E. Alcouffe)

This project involves the development and testing of computational methods for two-dimensional radiation hydrodynamics problems. As reported previously, ${ }^{7}$ a discretized version of a multifrequency $S_{N}$ radiative transfer equation coupled to an energy balance equation for the medium has been incorporated into a version of the TWODANT code. ${ }^{2}$ The $S_{N}$ solution is computed in each time step fully implicitly in the intensity. The required iterations 


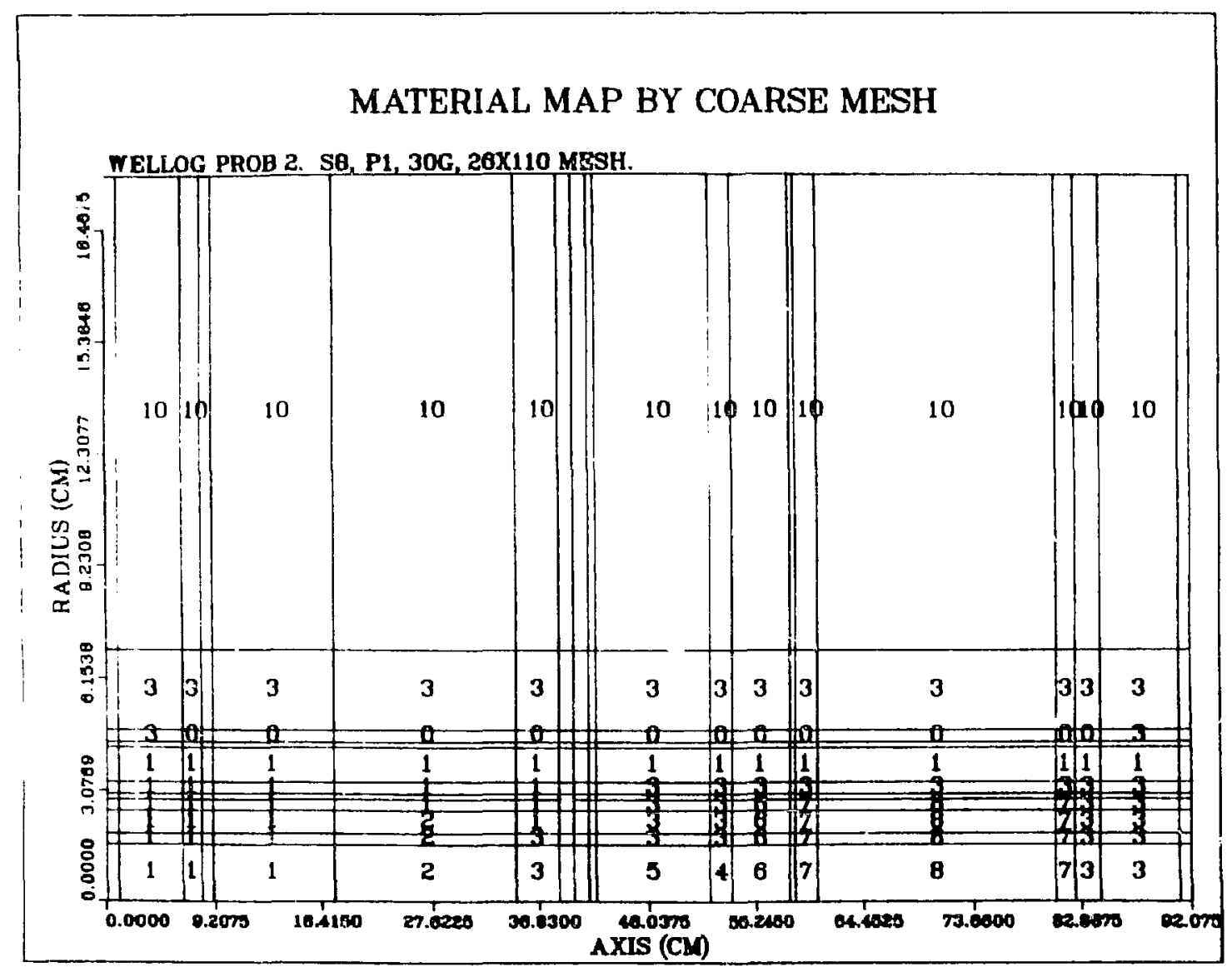

$\omega$

$\therefore$. 3. Prcblem used to test source corrention method of diffusion synthetic acceleration. 
are accelerated by the diffusion-corrected mult:group diffusion-syntheticacceleration method, which is in turn accelerated by a grey equation, that is, an equivalent one-group equation. The techniques used to discretize the procedure on an Eulerian grid are outlined in Ref. 7 . We have made one change in this, namely in the equation which computes the mesh-averaged emission rate from the vertex quantities. We now use the following equation:

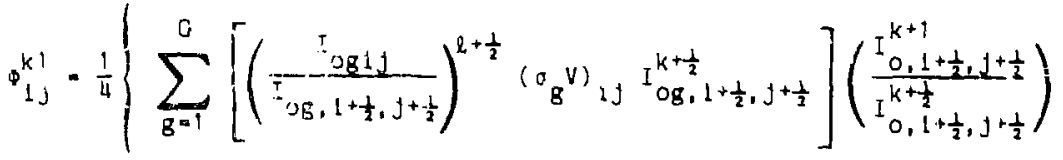

$$
\begin{aligned}
& +\sum_{B=1}^{G}\left[\left(\frac{I_{\text {Og } 1 J}}{I_{O B}, 1-\frac{1}{2}, J+\frac{1}{2}}\right)^{l+\frac{1}{2}}\left(\sigma_{B} V\right)_{1 J} I_{O B, 1-\frac{1}{2}, j+\frac{1}{2}}^{k+\frac{1}{2}}\right]\left(\frac{I_{0,1-\frac{1}{2}, j+\frac{1}{2}}^{k+1}}{I_{0,1-\frac{1}{2}, j+\frac{1}{2}}^{k+\frac{1}{2}}}\right) \\
& \cdot \sum_{g=1}^{G}\left[\left(\frac{I_{O g 1 J}}{I_{0 g}, 1+\frac{1}{2}, J-\frac{1}{2}}\right)^{l+\frac{1}{2}}\left(\sigma_{g} V\right)_{1 j} I_{0 g, I+\frac{1}{2}, J-\frac{1}{2}}^{k+\frac{1}{2}}\right]\left(\frac{I_{0,1+\frac{1}{2}, J-\frac{1}{2}}^{k+1}}{I_{0,1+\frac{1}{2}, J-\frac{1}{2}}^{k+\frac{1}{2}}}\right)
\end{aligned}
$$



where the quantities with superscripts $2+\frac{1}{2}$ refer to those from the transport iteration, the guantities with the superscript $k+\frac{1}{2} r \in f e r$ to the multigroup diffusion iteration, the quantities with superscript $k+1$ refer to the grey diffusion (one group) calculation, $\Phi_{i j}^{k+1}$ is the cell-centered emission density. $I_{o g}$ is the radiation intensity, and $\sigma_{g i j}$ is the emission opacity.

In using Eq. (2) we are maintaining compatibility with the discretized trarsport solution while providing a method for computing the accelerated emission source in cell ( $i j$ ) from the mesh corner quantities $I_{0, i \pm \frac{1}{2}, j \pm \frac{1}{2}}$.

As a test we compute a djfficult but not too complicated configuration with the existing code. The problem is a closed pipe with a disk barrier halfway from the source to the top end. A schematic is shown in Fig. 4, which shows the walls and barrier to be $1 \mathrm{~cm}$ thick; the source is at the left side, modeled as a 1-KeV Planckian, distributed uniformly over the nonwalled surface. The opacity for the interior of he pipe is given by the analytic expression 


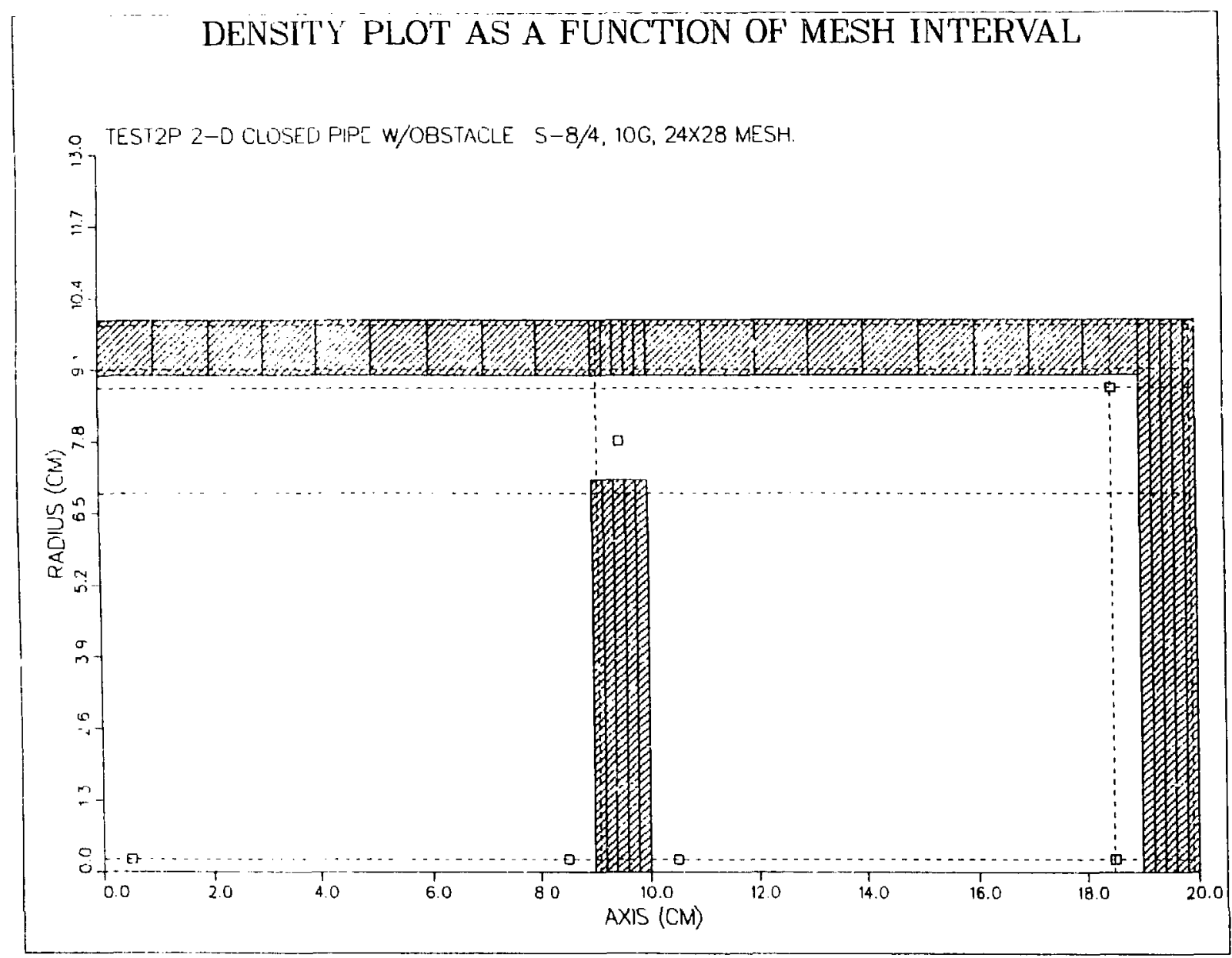

Fig. 4. Closed pipe with disk barrier model problem. 


$$
\sigma(\nu, T)=\frac{27\left(1-e^{-h \nu / k T}\right)}{(h v)^{3}} \mathrm{~cm}^{-1} \text {. }
$$

where hu and $\mathrm{kT}$ are in $\mathrm{KeV}$.

The opacity for the pipe walls is the same as Eq. (3) multiplied by 100 and for the barrier multiplied by 1000. This problem is run by switching the source on at $t=0$ and following the progress of the wave through the problem. The discretization used is 10 groups, $24 \times 28$ mesh, $S_{8}$ in groups 1 and 2 , and $S_{4}$ in groups 3 through 10. In Fig. 5 we show the time step as a function of time and in Fig. 6 we show the cumulative transport and diffusion iteration counts as a function of time. The calculation took 253 time steps, with 488 transport iterations and 1245 diffusion iterations in $331 \mathrm{CPU}$ sec. As seen in Fig. 5 the time step increases as the problem progresses from an initial step of $1.7 \times 10^{3} \mathrm{~s}$ to $3.3 \times 10^{-9} \mathrm{~s}$. The time step control is that the time step is increased by a factor of 1.5 if $\max _{i, j}\left|T_{i j}^{n+1}-T_{i j}^{n}\right| / T_{i j}^{n} \leq 0.05$ and decreased if the above exceeds 0.1 . If the iterations become ill-behaved, the time step is reduced by a factor of 5 to recover and preserve the calculation. Such an event can be seun in Fig. 5 at about $29 \mathrm{~ns}$. However, for this problem the computation is stable and successful up to the steady t tate configuration. In Figs. 7 and 8 we present the calculated spatial shapes of the axial temperature distribution at the specified times. Figure 7 is representative of a wave passing through a barrier, and Fig. 8 is representative of a wave moving down a channel. In Figs. 9a-9d, we present contour plots of the temperature at selected times to show the complicated history of the development from the initial state to the final state. This is also meant to demonstrate the robustness of the method in computing thermal wave problems in nontrivial geometries.

The accuracy of the solution is, of course, a concern. We assess the discretized time accuracy of the solution by reducing the time step criterion and tightening up on the iteration convergence criteria. We did this in two steps: in the first case, we set the time step criterion to 0.05 (from 0.1 ) and the convergence criterion to 0.005 (from 0.01); in the second case, we set the time step criterion to 0.02 and the convergence criterion to 0.003 . In Fig. 10 we present a comparison calculation of the temperature at selected mesh points as a function of time for the original case and the first refinement. It is seen that the original case is fairly well converged. When the first and 


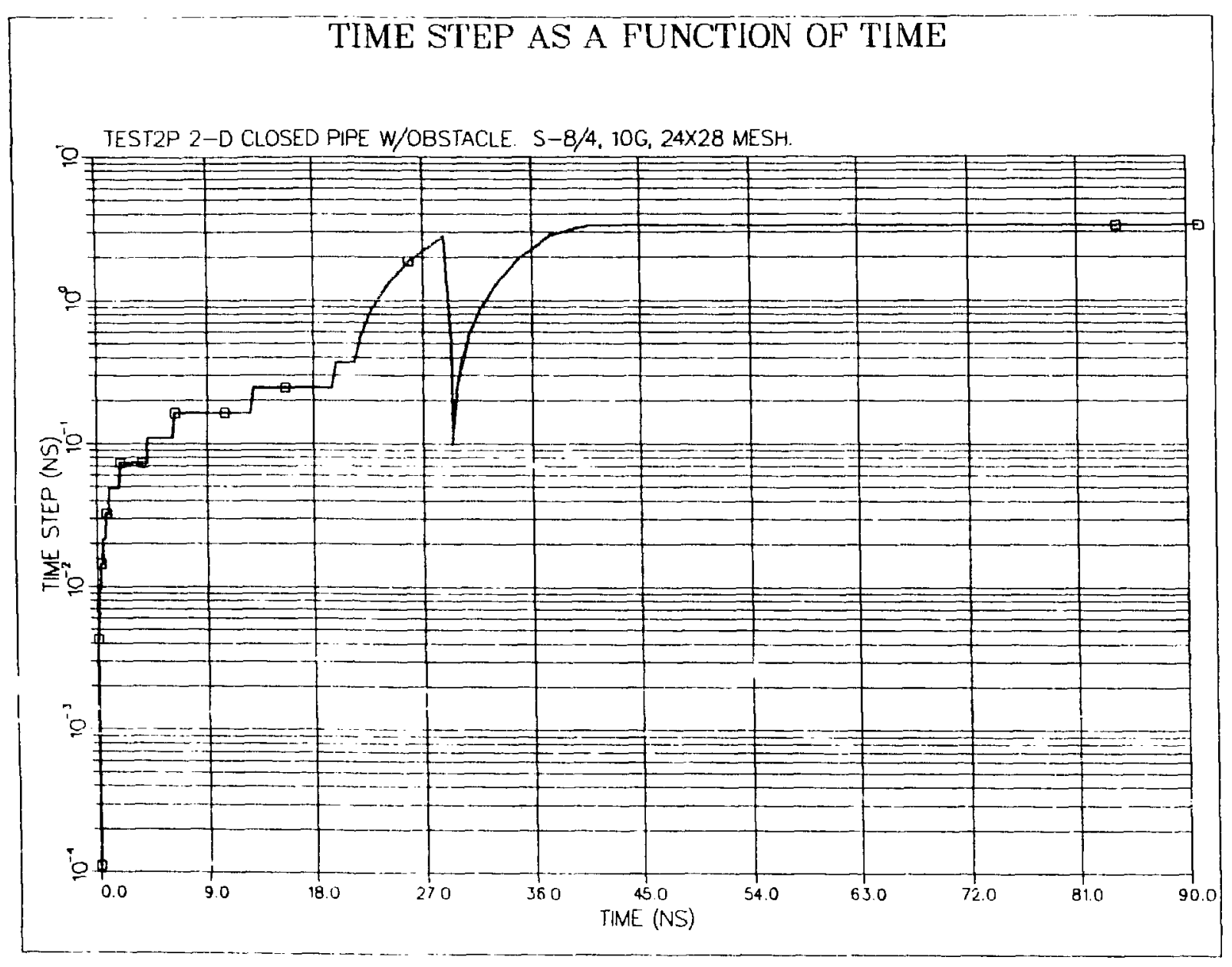

Fig. 5. Time step as a function of time for model problem. 


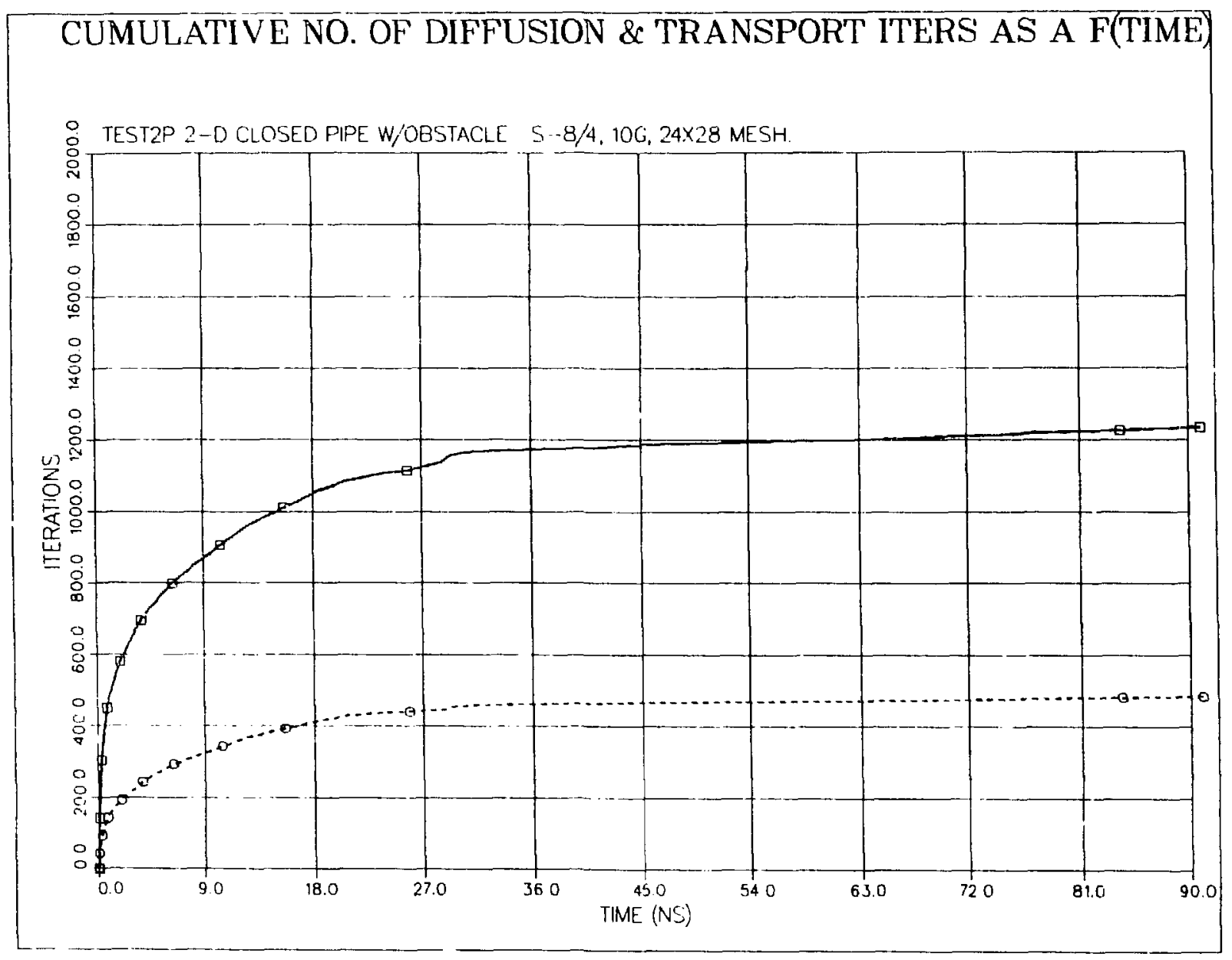

Fig. 6. Cumulative number of diffusion and transport iterations as a function of time. 


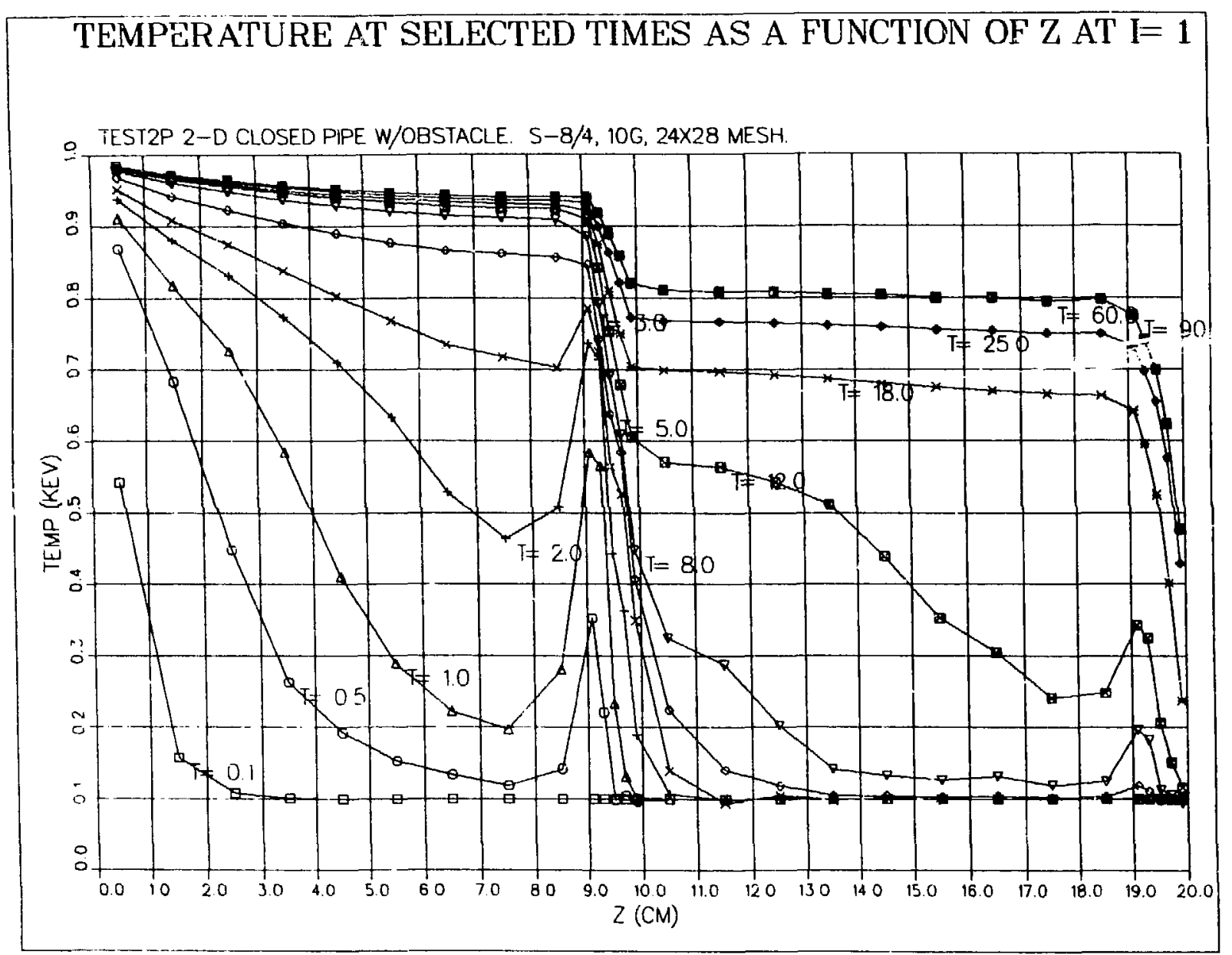

Fig. 7. Temperature at selected times as a function of $z$ for fixed $r(I=1)$. 


\section{TEMPERATURE AT SELECTED TMASS AS A FUNCTION OF Z AT I=19}

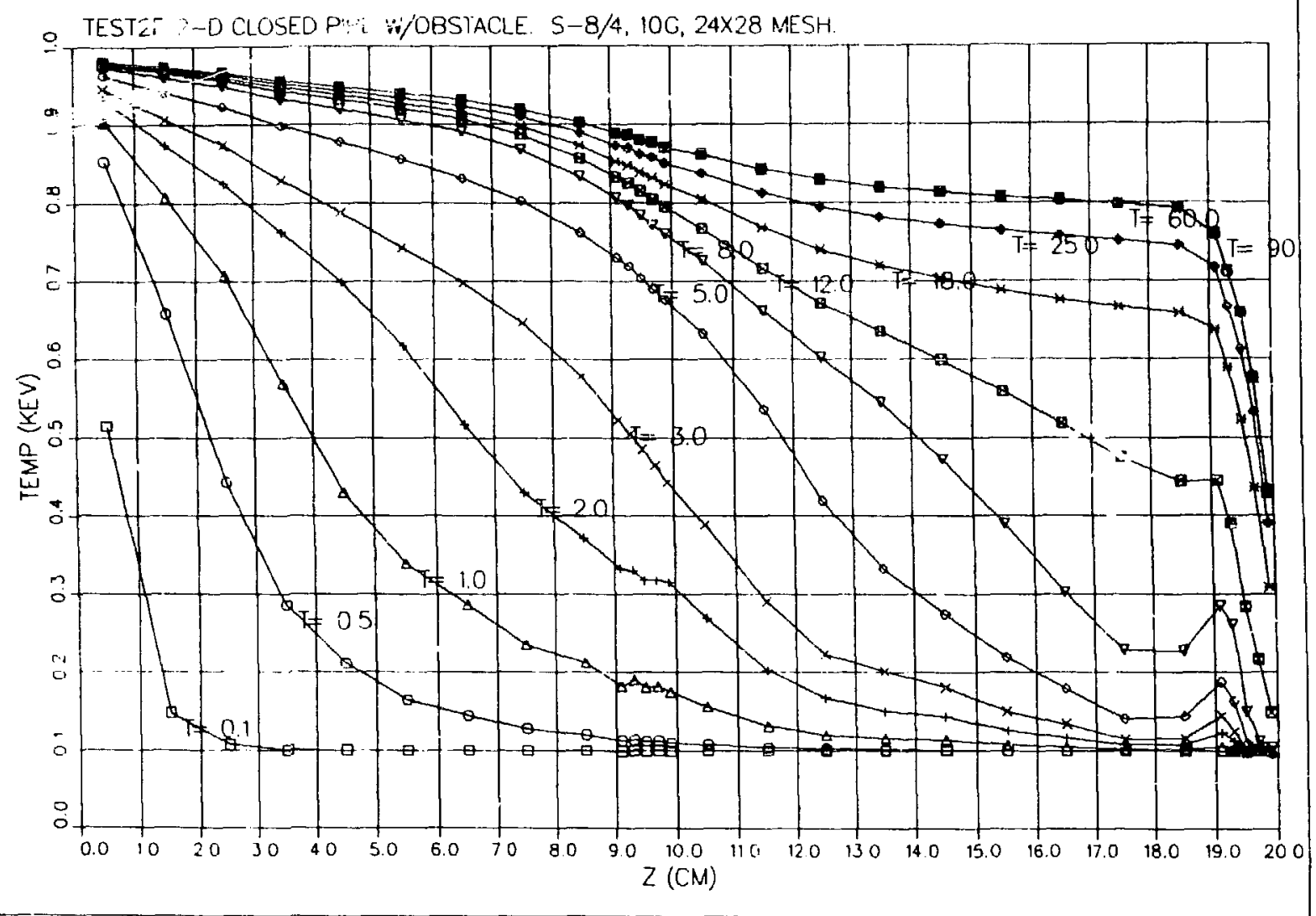

Fig. 8. Temperature at selected times as a function of $z$ for fixed $r(I=19)$. 


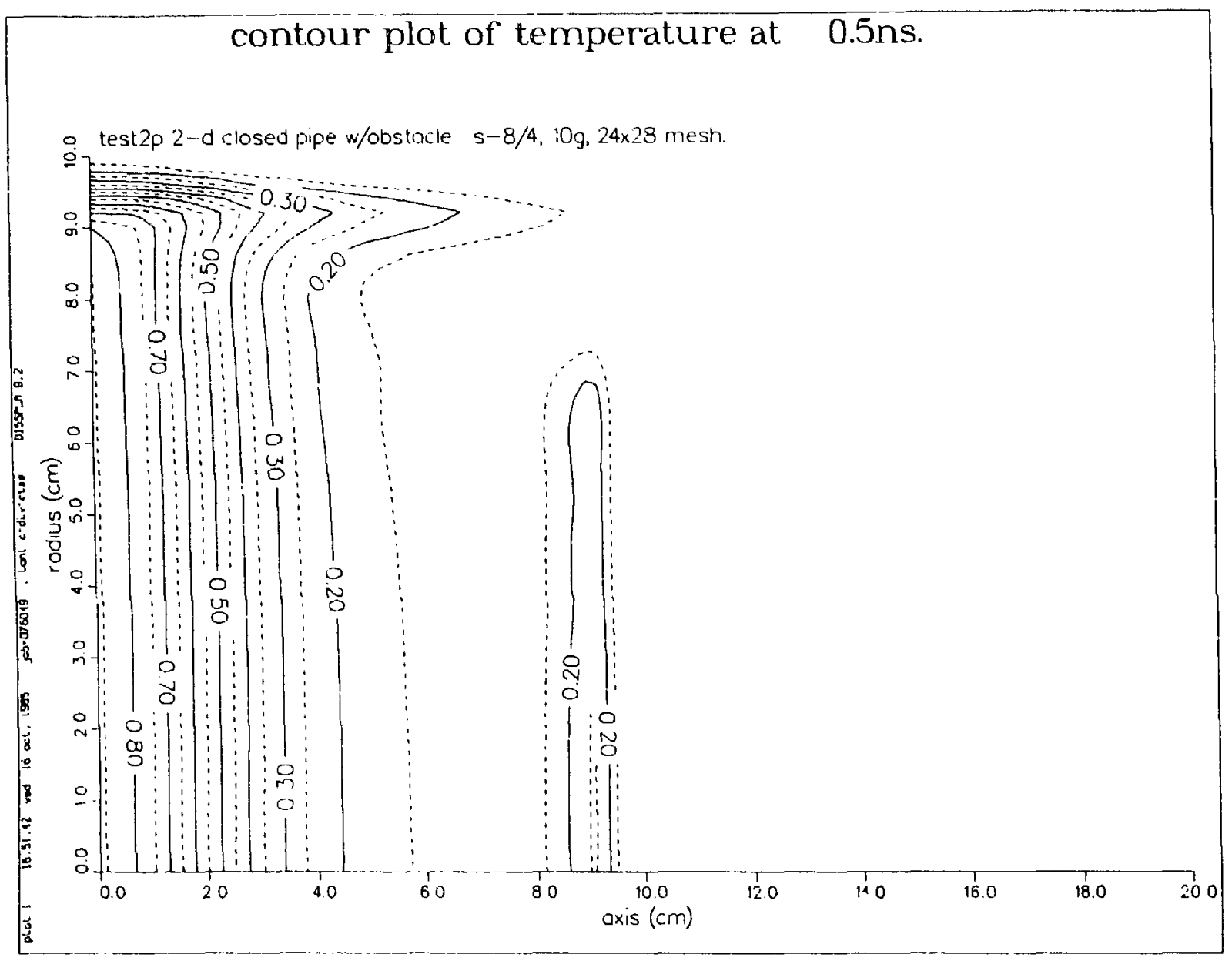

F.g. 9a. Contour plot of temperature at $0.5 \mathrm{~ns}$. 


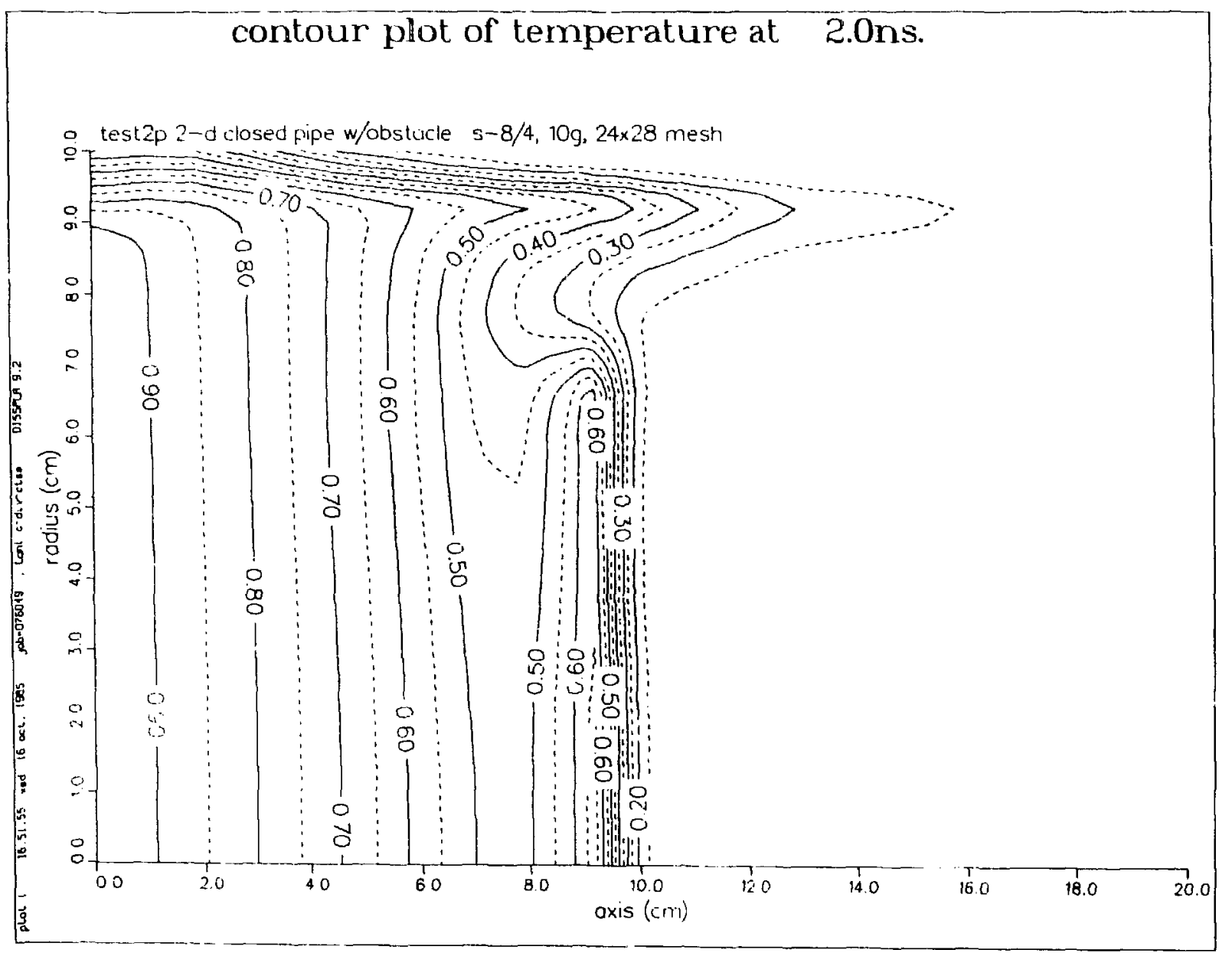

Fig. 9b. Contour plot of temperature at $2.0 \mathrm{~ns}$. 


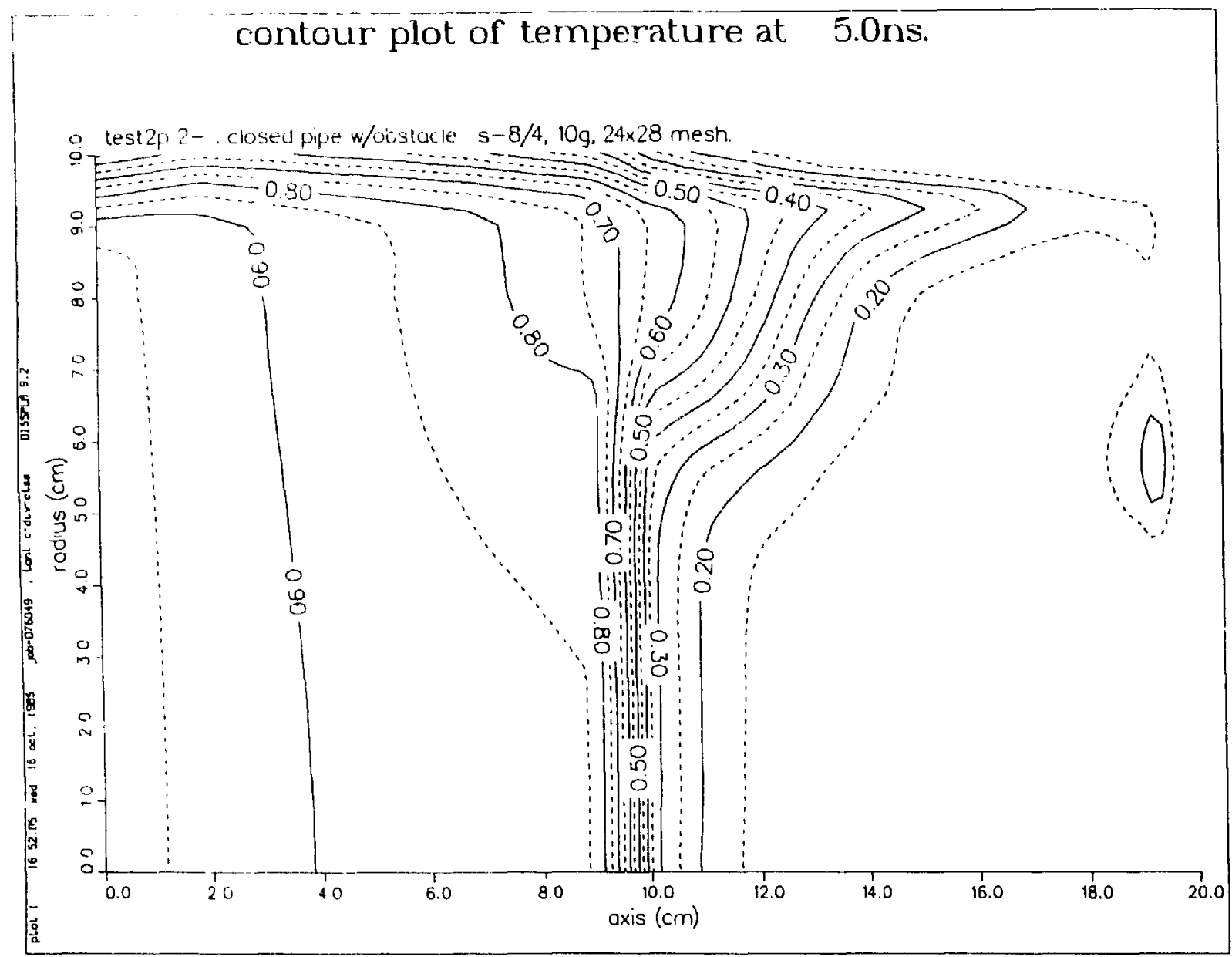

Fig. 9c. Contour plot of temperature at $5.0 \mathrm{~ns}$. 


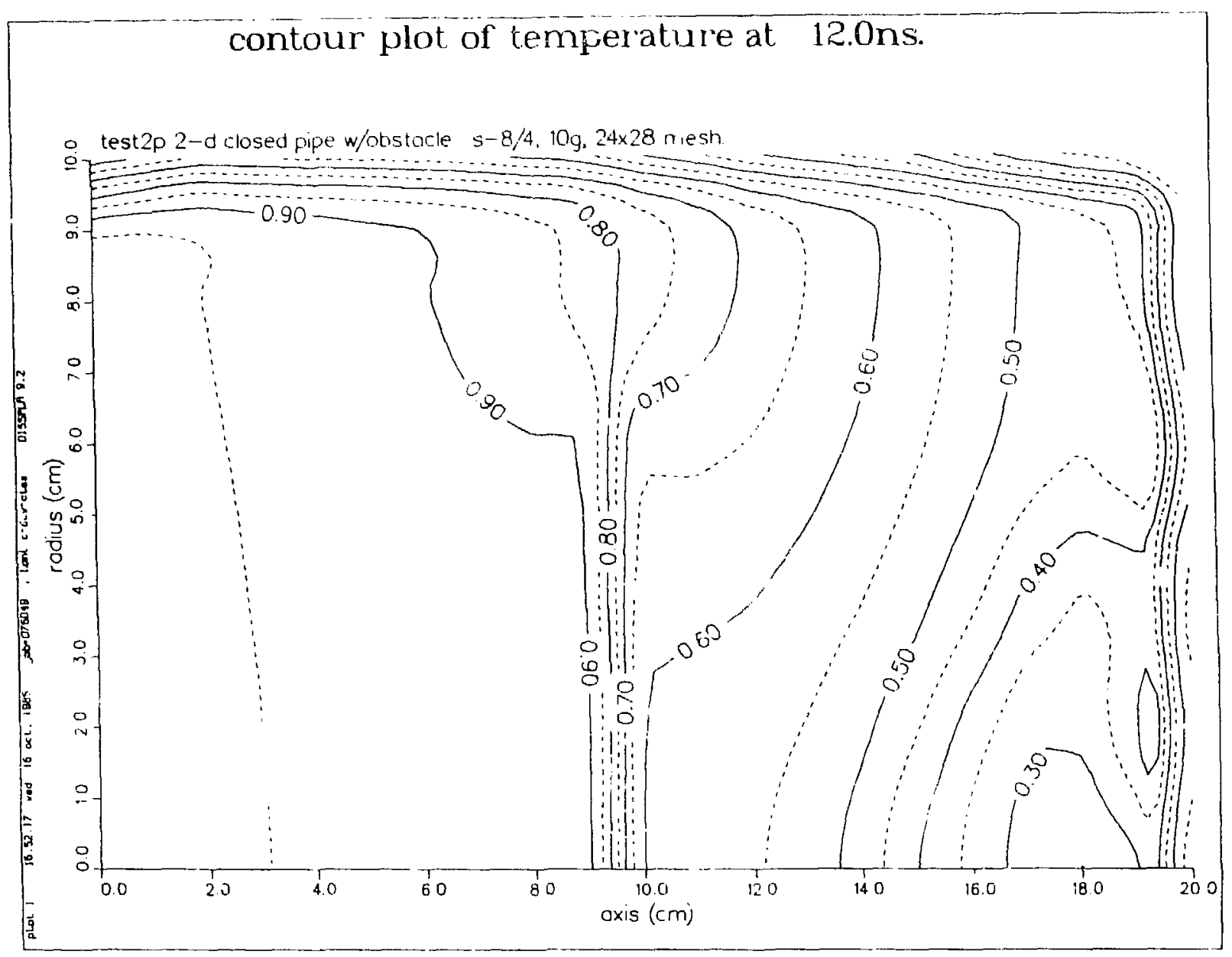

Fig. 9d. Contour plot of temperature at $12.0 \mathrm{~ns}$. 


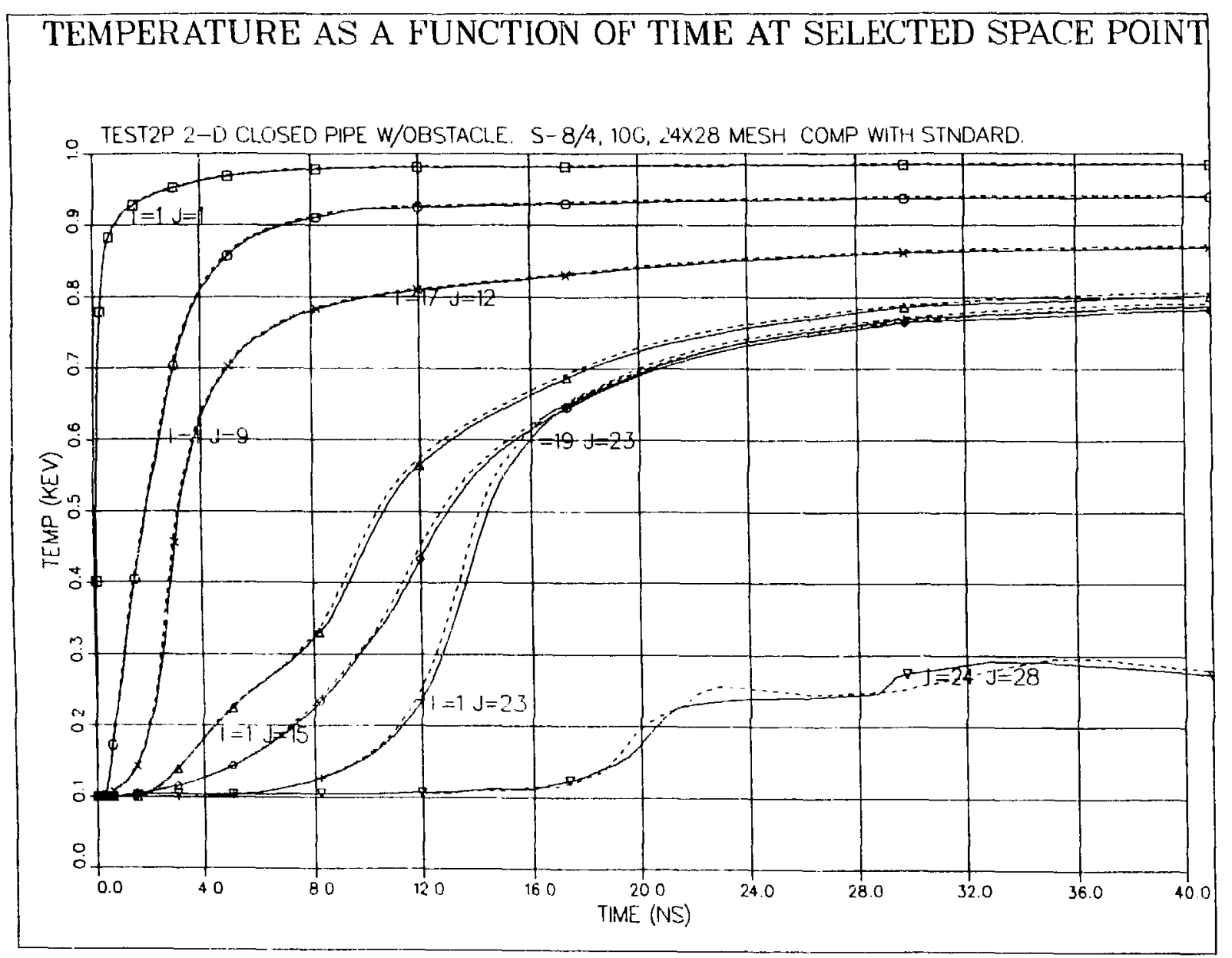

Fig. 10. Comparison of temperature distribution for standard case. (solid line) and first refinement of time step and convergence criterion (dashed line). 
second refinements are compared, they yield nearly identical results. Thus, it seems that the calculational procedure ylelds an accurate result for what we term the nominal convergence criteria. This does not address the accuracy of the other discretizations, which will be the object of another study.

F. Asymptotic Solutions of Numerical Transport Problems in Diffusive Regimes (E. W. Larsen, and W. F. Miller, Jr.)

In considering numerical methods for deterministically solving radiative transfer problems, one must typically face the situation that in the highfrequency groups the spatial meshes are optically thin, but in the lowfrequency groups the spatial meshes are optically thick. Since standard error analyses of numerical transport schemes generally predict errors which are small for optically thin cells and which grow as the optical thickness grows, there is strong reason to doubt the validity of numerical results which are obtained for optically thick meshes. However, for many applications in radiative transfer and neutron transport, the problems in which optically thick cells occur are also ones for which the problem is "diffusive," thai is, gradients are small and the solution depends weakly on angle. For these problems one generally expects the solution of a diffusion equation to be an accurate approximation to the solution of the transport equation. In fact, asymptotic theory already exists ${ }^{8}$ which describes the transition from transport to diffusion theory for such problems. We have applied this same asymptotic expansion to various discretized transport equations to determine whether, in the same asymptotic limit, these discretizations lead to legitimate discretizations of the appropriate diffusion equation, in the hope of determining wrether reasonable numerical results can be obtained for problems with optically thick spatial cells. In other words, we expect that 1 i a transport differencing scheme does become a legitimate diffusion differencing scheme in this asymptotic limit, one should expect good numerical results ( $1 \mathrm{f}$ the diffusion scheme itself is itself adequately differenced), and conversely, if the transport scheme does not transition into a diffusion scheme, one should not expect good numerical results.

The specific transport schemes we have studied involve two types of unknown quantities per cell: the cell average and cell edge fluxes. (We adopt the terminology of neutron transport in this discussion.) It is legitimate to 
ask whether either or both of these quantities satisfy a diffusion limit. Also, we have considered two separate types of spatial meshes,

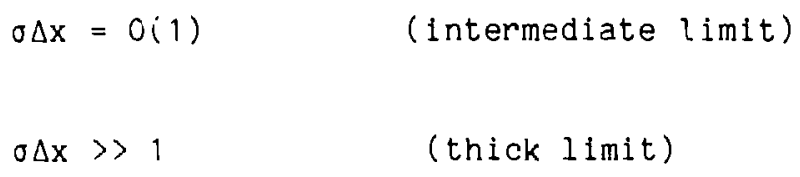

which describe problems with spatial cells which are not optically small. (If $\sigma \Delta x \ll 1$, standard truncation analyses are applicable and show that the correct diffusion limit is attained because the formal truncation errors are negligible.) Thus, for each scheme, we have a total of four separate asymptotic limits: the intermediate and thick ilmits for the cell edge and cell average fluxes.

We have considered five differencing schemes applicable in neutron transport or radiative transport applications: the Diamond, 9 step, Lund-Wilson: 10 Castor, 11 and a "new" differencing scheme. These schemes all have the balance equation as a common ingredient,

$$
\frac{\mu_{m}}{\Delta x_{j}}\left(\psi_{m, j+\frac{1}{2}}-\psi_{m, j-\frac{1}{2}}\right)+\sigma_{T j} \psi_{m j}=\sigma_{s j} \sum_{n=1}^{N} \psi_{n j} w_{n}+Q_{j}
$$

and are all distinguished by the choice of auxiliary equation. The we.l-known Diamond and Step auxiliary equations ${ }^{9}$ are

$$
\begin{array}{ll}
\psi_{m j}=\frac{1}{2}\left(\psi_{m, j+\frac{1}{2}}+\psi_{m, j-\frac{1}{2}}\right) & \text { (Di amond) , } \\
\psi_{m j}=\psi_{m, j \pm \frac{1}{2}} & \text { (Step) , }
\end{array}
$$

where here and in the following we interpret " \pm " to mean "+" if $\mu_{m}>0$ and "-" if $\mu_{m}<0$. The Lund-Wilson auxiliary equation ${ }^{10}$ is

$$
\psi_{m j}=\gamma_{m, j \pm \frac{1}{2}} \psi_{m, j \pm \frac{1}{2}}
$$

where 


$$
\gamma_{m, j+\frac{1}{2}}=1+\tau_{m, j+\frac{1}{2}}
$$

and

$$
\tau_{m, j+\frac{1}{2}}=\frac{1}{\prod_{m} T}\left[\frac{1}{\left(\sigma_{T} \Delta x\right)_{j}}+\frac{1}{\left(\sigma_{T} \Delta x\right)_{j+1}}\right]^{-1} ;
$$

the Castor auxiliary equation ${ }^{11}$ is defined by Eqs. (7a), (7a), and

$$
\gamma_{m, j+\frac{1}{2}}=\frac{\tau m, j+\frac{1}{2}}{2}+\left[1+\left(\frac{{ }^{\tau} m, j+\frac{1}{2}}{2}\right)^{2}\right]^{\frac{1}{2}} .
$$

and the "new" auxillary equation is

$$
\begin{aligned}
& \psi_{m j}=\gamma_{m j} \psi_{m, j \pm \frac{1}{2}}+\left(1-\gamma_{m j}\right) \frac{\sigma_{s j} \xi_{j \pm \frac{1}{2}}+Q_{j}}{\sigma_{m j}}, \\
& \gamma_{m j}=1+\frac{\left(\sigma_{T} \Delta x\right)_{j}}{2\left|\mu_{m}\right|}, \\
& \xi_{j+\frac{1}{2}}=\frac{\left(\sigma_{T} \Delta x\right)_{j+1} \sum_{n=1}^{N} \psi_{n j} w_{n}+\left(\sigma_{T} \Delta x\right)_{j} \sum_{n=1}^{N} \psi_{n, j+1} w_{n}}{\left(\sigma_{T} \Delta x\right)_{j+1}+\left(\sigma_{T} \Delta x\right)_{j}} .
\end{aligned}
$$

The asymptotic expansion ${ }^{8}$ begins by introducing the scalings

$$
\begin{aligned}
& \sigma_{T} \rightarrow \frac{\bar{\sigma}_{T}}{\varepsilon}, \\
& \sigma_{S} \rightarrow \frac{\bar{\sigma}_{T}}{\varepsilon}-\varepsilon\left(\bar{\sigma}_{T}-\tilde{\sigma}_{S}\right), \\
& Q \rightarrow \varepsilon \bar{Q},
\end{aligned}
$$

and

$$
\Delta x_{j} \rightarrow\left\{\begin{array}{cc}
\varepsilon \overline{\Delta x}_{j} & \text { (intermediate limit) } \\
\tilde{\Delta x}_{j} & \text { (thick limit) }
\end{array}\right.
$$


where the tilde quantities on the right sides of the arrow are $0(1)$, and $\varepsilon$ is a small dimensionless parameter. Then we expand the unknown quantities in the numerical schemes in a power series in $\varepsilon$. The results of this analysis are indicated below in Table I, where "yes" means that the appropriate limit is satisfied, "no" means it is not, and "maybe" is a qualifier indicating that under special conditions the limit is satisfied, but in general it is not.

These results indicate some of the good and bad features of each method in the various asymptotic regimes. In particular, the diamond scheme can be expected always to produce cell average fluxes which satisfy the correct diffusion in the thick diffusion limit, but this is not true for the cell edge fluxes. Also, the step method can "almost always" be expected to produce poor results in diffusive problems, as is consistent with conventional wisdom regarding this method. The Lund-Wilson and Castor methods will always produce poor cell edge fluxes, and so in any problem which is driven by a boundary source, or where material boundaries play an important role, these methods can be expected to be inaccurate. The "new" method is included to show that it is possible for a differencing scheme to possess all four diffusion limits.

We have tested our theoretical results on several problems which were selected to check all of the predictions of the theory, and we have found that

TABLE I

SUMMARY OF RESULTS

\begin{tabular}{|c|c|c|c|c|}
\hline & \multicolumn{2}{|c|}{ Intermediate } & \multicolumn{2}{|c|}{ Thick } \\
\hline & Edge & Average & Edge & Average \\
\hline$D$ lamond & yes & & maybe & \\
\hline Step & maybe ${ }^{\circ}$ & maybe & maybe & maybe \\
\hline Lund-Wilson & no & maybe & no & yes \\
\hline Castor & no & maybe & no & yes \\
\hline New & yes & yes & yes & yes \\
\hline
\end{tabular}

Quallflers are defined as follows:

$a_{Y e s}$ only if the boundary sources are 1sotroplc.

$b_{Y e s}$ only if $\sigma_{a j}=Q_{j}=0, \sigma_{T j}=$ constant, and $\Delta x_{j}=$ constant.

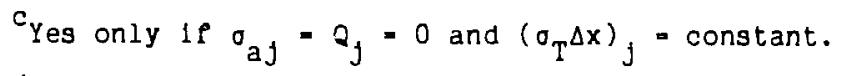

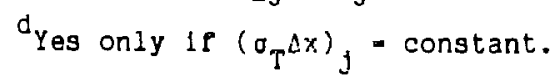


in every case the numerical results agree with the theoretical predictions. The details of the analysis and the numerical experimentations are much too lengthy to include here, but they are included in a full paper which has been prepared for publication. 12

In conclusion, we expect that the asymptotic analysis which we have developed will play an important role in determining the behavior and accuracy of transport differencing schemes in diffusive systems with optlcally thick cells.

G. Maximal Principles for Radiative Transfer Problems (E. W. Larsen)

In the absence of material motion, heat conduction, and internal sources, the equations of radiative transfer can be written

$$
\begin{aligned}
& \frac{1}{c} \frac{\partial I}{\partial t}+\underline{\Omega} \cdot \underline{\nabla}=\sigma(B-I), \\
& c_{v} \frac{\partial T}{\partial t}=\iint \sigma(I-B) d v d \Omega,
\end{aligned}
$$

with initial conditions

$$
\begin{aligned}
& I(\underline{x}, \underline{\Omega}, v, 0)=I_{i}(\underline{x}, \underline{\Omega}, v), \\
& T(\underline{x}, 0)=T_{1}(\underline{x})
\end{aligned}
$$

and boundary conditions

$$
I(\underline{x}, \underline{\Omega}, v, 0)=I_{b}(\underline{x}, \underline{\Omega}, v), \quad \underline{x} \varepsilon \partial D, \underline{\Omega} \cdot \underline{r}<0 .
$$

The notation in these equations is standard: ${ }^{13} I(\underline{x}, \underline{\Omega}, v, t)$ is the specific intensity of radiation at $\underline{x} \in D(D=$ spatial domain) traveling in the direction $\underline{\Omega}$ (with $|\underline{\Omega}|=1$ ) at frequency $v(0<v<\infty)$ at $t$ ime $t ; T(\underline{x}, t)$ is the temperature; $c$ is the speed of light; $\sigma(v, t)$ is the opacity; $B(v, T)$ is the Planck function

$$
B(v, T)=\frac{2 h}{c^{2}} \frac{3^{3}}{e^{h v / k T}-1} \text {, }
$$

$h$ is Planck's constant; and $k$ is Boltzmann's constant. We have 


$$
\iint B d v d \Omega=a c T^{4} \equiv \phi
$$

where

$$
a=\frac{8 \pi^{5} k^{4}}{15 h^{3} c^{2}}
$$

is the radiation constant, and $\phi=\phi(\underline{x}, t)$ is the equilibrium energy density. Also, we note that $B$ is a strictly increasing function of $T$; that is, if $T_{1}$ $<T_{2}$, for all $\nu$ we have $B\left(\nu, T_{1}\right)<B\left(\nu, T_{2}\right)$.

Until recently, little was known about the properties of the solution of problem (11), or even whether a solution exists. However, Mercier ${ }^{14}$ (and, in a follow-up paper, Golse and Perthame ${ }^{15}$ ) have recently proved, under some technical constraints on $\sigma$ which are not always met in actual problems, that a unique solution of problem (11) exists. Moreover, they have shown that if a solution exists, it satisfies the following:

Maximum Principle: Let there exist constant temperatures $0<T_{L}<T_{U}$ such that

$$
\begin{aligned}
& B\left(v, T_{L}\right)<I_{i}(\underline{x}, \underline{\Omega}, v)<B\left(v, T_{U}\right), \\
& T_{L}<T_{i}(\underline{x})<T_{U},
\end{aligned}
$$

and

$$
B\left(v, T_{L}\right)<I_{D}(\underline{x}, \underline{\Omega}, v)<B\left(v, T_{U}\right) .
$$

Then [for all $\underline{x} \in D,|\underline{\Omega}|=1, v>0$, and $t>0]$

$$
\begin{aligned}
& B\left(v, T_{L}\right)<I(\underline{x}, \underline{\Omega}, v, t)<B\left(v, T_{U}\right), \\
& T_{L}<T(\underline{x}, t)<T_{U} .
\end{aligned}
$$

Mercier's proof relies on numerous integral inequalities. However, we have devised a separate proof which is conceptually very simple, which makes no unphysical assumptions on the opacities, and which we have applied to Monte 28 
Carlo and ceterministic numerical methods for problem 11 to determine whether bounds on the time step $\Delta t$ exist for these methods which guarantee that the numerical solutions satisfy the maximum principle. In the following, we give a sketch of our proof of the maximum princlple; ther we summarize the results obtained by applying a similar analysis to the numerical methods. The details of this analysis are straightforward but lengthy, and we will present them elsewhere.

We now state the assumptions which we require for our proof of the maximum principle. We assume that a solution of problem 11 exists and that for each $\underline{x}, \phi$ is a continuous function of $t$. [If $I_{i}, I_{b}$, and $\phi_{1}$ are piecewisecontinuous functions of their variables, then owing to the structure of Eq. (11a) it is reasonable that I will be piecewise continuous, and thus, by the structure of ( $11 \mathrm{~b})$, that $\phi$ will be continuous in $t$. Thus, the assumption that $\phi$ is continuous in $t$ is mathematically reasonable.]

Given these assumptions, our proof of the maximum principle is as follows. First, we solve Eqs. $(11 a, 0, e)$ for I to obtain

$$
\begin{aligned}
I(\underline{x}, \underline{\Omega}, v, t)= & I_{0}(\underline{x}, \underline{\Omega}, v, t) \exp \left\{-\int_{0}^{\rho(\underline{x}, \underline{\Omega}, t)} d[v, T(\underline{x}-\underline{s}, t-s / c)] d s\right. \\
& +\int_{0}^{\rho(\underline{x}, \underline{\Omega}, t)}(\sigma B)[v, T(\underline{x}-\underline{\Omega} s, t-s / c)] \exp \left\{-\int_{0}^{\rho(\underline{x}, \underline{\Omega}, t)} \sigma\left[v, T\left(\underline{x}-s^{\prime} \underline{\Omega}, t-s^{\prime} / c\right)\right] d s .\right.
\end{aligned}
$$

Here

$$
I_{0}(\underline{x}, \underline{\Omega}, t)= \begin{cases}I_{b}\left(\underline{x}-\rho \underline{\Omega}, \underline{\Omega}, v, t-\frac{\rho}{c}\right), & \ell(\underline{x}, \underline{\Omega})<c t, \\ I_{1}(\underline{x}-\rho \underline{\Omega}, \underline{\Omega}, v), & \text { ct }<\ell(\underline{x}, \underline{\Omega}), \\ \rho(\underline{x}, \underline{\Omega}, t)=\min [\mathrm{ct}, \ell(\underline{x}, \underline{\Omega})], & \end{cases}
$$

and $\ell(\underline{x}, \underline{\Omega})$ is the distance from $\underline{x}$ to $\partial D$ in the direction of $\underline{\underline{\Omega}}$. This formal solution for I was obtalned in a straightforward manner using the method of characteristics. In words, the characteristic line ( $x-s \underline{\Omega}, t-s / c)$ emanating backward from the point $(\underline{x}, t)$ intersects the initial time $(t=0)$ if $s=c t<$ $\ell(\underline{x}, \underline{\Omega})$, or it intersects the boundary $(\partial D)$ if $s=\ell(\underline{x}, \underline{\Omega})<c t$. In either case, 
the length of this line (that is, the appropriate value of $s$ ) is $\rho(\underline{x}, \underline{\Omega}, t)$, and the known value for I at the starting point $(\underline{x}-p \underline{\Omega}, t-\rho / c)$ is $I_{0}{ }^{\cdot}$ we note that Eqs. (15a), (15c) and (18) imply

$$
B\left(v, T_{L}\right)<I_{0}(\underline{x}, \underline{\Omega}, v, t)<B\left(v, T_{U}\right)
$$

Next, we introduce Eq. (17) into Eq. (11b) to obtain the following single equation for $\mathrm{T}$ :

$$
c_{v} \frac{\partial T}{\partial t}=\quad \sigma\left[I_{0} e^{-\int^{\rho} \sigma d s}+\int_{0}^{\rho}(\sigma B)(s) e^{-\int^{\rho} \sigma d s^{\prime}} d s-B\right] d u d \Omega
$$

Using the inequalities (20) and the Identity

$$
e^{-\int_{0}^{\rho} \sigma d s}=1-\int_{0}^{\rho} \sigma(s) e^{-\int_{0}^{s} \sigma\left(s^{\prime}\right) d s^{\prime}} d s
$$

we easily get from (21)

$$
\begin{aligned}
& \frac{\partial T}{\partial t}<\frac{1}{c_{v}} \iint \sigma\left\{\left(B_{U}-B\right)-\int_{0}^{\rho}\left[\sigma\left(B_{U}-B\right)\right](s) e^{-\int_{0}^{s} \sigma d s^{\prime}} d s\right\} d v d \Omega \text {, } \\
& \frac{\partial T}{\partial t}>\frac{1}{c_{v}} \iint \sigma\left\{\left(B_{L}-B\right)+\int_{0}^{\rho}\left[\sigma\left(B-B_{L}\right)\right](s) e^{-\int^{s} \sigma d s^{\prime}} d s\right\} d v d \Omega,
\end{aligned}
$$

where $B_{U}=B\left(v, T_{U}\right)$, etc.

The remainder of the proof consists of two parts. First, we shall use the inequalities (23) to derive (16b), and then we shall use (16b) to derive $(16 \mathrm{a})$.

To derive (16b), we give a proof by contradiction. Thus, suppose (16b) is not satisfied for all time. Then there exists a time $t_{0}$ and a point $x_{0} \varepsilon D$ such that 


$$
\begin{aligned}
& T\left(\underline{x}_{0}, t_{0}\right)=T_{U} \\
& {\left[\operatorname{or} T\left(\underline{x}_{0}, t_{0}\right)=T_{L}\right]}
\end{aligned}
$$

and

$$
T_{L}<T(\underline{x}, t)<T_{U}, \quad \text { all } \underline{x} \in D, 0 \leq t<t_{0} .
$$

For definiteness, let us suppose that (24a) holds. Then the inequality (23a) gives

$$
\begin{aligned}
& \frac{\partial}{\partial t} T\left(\underline{x}_{0}, t\right)<\frac{1}{c_{v}\left[T\left(\underline{x}_{0}, t\right)\right]} \iint 0\left[v, T\left(\underline{x}_{0}, t\right)\right]\{B(v, T U \\
&\left.-B\left[v, t\left(\underline{x}_{0}, t\right)\right]\right\} d v d \Omega, \quad 0<t<t_{0} .
\end{aligned}
$$

However, if we define the constant $M$ by

$$
M=T_{L}<T<T_{U} \frac{4 \pi}{c_{v}(T)} \int \sigma(\nu, T) \frac{B\left(\nu, T_{U}\right)-B(\nu, T)}{T_{U}-T} d v
$$

then (26) implies

$$
\frac{\partial}{\partial t} T\left(\underline{x}_{0}, t\right)<M\left[T T_{U}-T\left(\underline{x}_{0}, t\right)\right], \quad 0 \leq t \leq t \underline{t}_{0}
$$

This result and (15b) then give

$$
T\left(\underline{x}_{0}, t_{0}\right)<T_{U}-\left[T_{U}-T_{i}\left(\underline{x}_{0}\right)\right] e^{-M t_{0}}<T_{U}
$$

which contradicts ( $24 \mathrm{a}$ ). Likewise, ( $24 \mathrm{~b}$ ) also leads to a contradiction. Hence, the inequalities (160) are satisfied for all time (or, to be more precise, for as long as our basic assumptions are satisfied).

We now have

$$
B\left(\nu, T_{L}\right)<I_{0} \text {, and } B(\nu, T)<B\left(\nu, T_{U}\right) \text {, }
$$


and introducing these inequalities and the identity (22) into Eq. (17), we immediately get

$$
B\left(U, T_{L}\right)<I<B\left(U, T_{U}\right)
$$

This completes the proof of the maximum principle.

Next we shall summarize the results which we have obtained by applying a similar type of analysis to (time-discretized) numerical schemes for solving problem (11). Specifically, we consider the Fleck-Cummings ${ }^{16}$ (FC) scheme, which is the basis of much implicit Monte Carlo work, and three deterministic schemes, one of which is based directly on the FC scheme.

The FC scheme arises from linking a time-discretized version of Eq. (11b) with a version of Eq. (11a) in which time is not discretized. Here one does not use the temperature $T(\underline{x}, t)$ as the second basic unknown, but rather the equilibrium energy density

$$
\phi(\underline{x}, t)=a \subset T^{4}(\underline{x}, t) .
$$

[See Eq. (13).] Accordingly, Eq. (11b) is rewritten as

$$
\frac{\partial \phi}{\partial t}=B(\phi) \iint \sigma(I-B) d \nu d \Omega,
$$

where 3 , which is determined by

$$
c_{v} \frac{\partial T}{\partial t}=\frac{1}{B} \frac{\partial \phi}{\partial t}
$$

is explicitly given by

$$
B(\phi)=4(\mathrm{ac})^{\frac{1}{4}} \frac{\phi^{\frac{3}{4}}}{c_{V}\left[(\phi / a c)^{\frac{1}{4}}\right]} .
$$

Also, in this scheme there is a "Fleck" parameter $\alpha$ which determines a degree of implicitness and which is to be chosen between $1 / 2$ and 1 . (The cholce $\alpha=1 / 2$ is supposed to be the most accurate, but $\alpha=1$ is the most stable.) Let us define the constants 


$$
\begin{aligned}
& \xi_{U}=\sup _{T_{L}<T<T_{U}} \frac{T}{T_{U}-T} \frac{\int \sigma(\nu, T)[B(\nu, T U)-B(\nu, T)] d \nu}{\int \sigma(\nu, T) B(\nu, T) d \nu}, \\
& \xi_{L}=\sup _{T_{L}<T<T_{U}} \frac{T}{T-T_{L}} \frac{\int \sigma(v, T)\left[B(\nu, T)-B\left(\nu, T_{L}\right)\right] d v}{\int \sigma(v, T) B(v, T) d v}, \\
& \eta_{U}=\sup _{T_{L}<T<T_{U}} \frac{T^{4}}{T_{U}^{4}-T^{4}} \frac{\int \sigma(v, T)\left[B\left(v, T_{U}\right)-B(v, T)\right] d v}{\int \sigma(v, T) B(v, T) d v}, \\
& \eta_{L}=\sup _{T_{L}<T<T_{U}} \frac{T^{4}}{T^{4}-T_{L}^{4}} \frac{\int \sigma(v, T)\left[B(v, T)-B\left(\nu, T_{L}\right)\right]}{\int_{\sigma}(v, T) B(v, T) d v}, \\
& Y=T_{L}<T<T_{U} \frac{4 T}{c_{v}(T)}-\frac{1}{\int_{\sigma}(v, T) B(\nu, T) d v} .
\end{aligned}
$$

Then, in terms of these known constants (which are all positive) and $\alpha$, we have the following result:

Maximum Principle for the Fleck-Cummings Method: If the time step $\Delta t$ satisfies

$$
\max \left(\alpha \xi_{U}, \alpha \xi_{L}, \eta_{U}, \eta_{L}\right)<\frac{\gamma}{\Delta t}+\alpha,
$$

then the Fleck-Cummings solution satisfies the Maximum Principle. [That is, if $\Delta t$ satisfies (36), and if the initial and boundary conditions satisfy (15), then the Fleck-Cummings $I$ and $\phi_{n}$ satisfy

$$
B\left(v, T_{L}\right)<I(\underline{x}, \underline{\Omega}, v, t)<B\left(v, T_{U}\right)
$$

and 


$$
\operatorname{act}_{L}^{4}<\Phi_{n}(x)<\operatorname{acT}_{U}^{4}
$$

for all n.]

Remarks: The choice $\alpha=1$, which is supposed to make the method the most stable, does not necessarily lead to a largest $\Delta t$ for which the maximum principle holds. In other words, setting $\alpha=1$ can lead to a smaller allowable $\Delta t$ than wolld occur if a were chosen less than one. Second, our result does not predict that the inequality (36) is sharp, in that if it is vio?ated, then the Fleck-Cummings solution will violate the maximum principle. However, it does show that if (36) is violated, the Fleck-Cummings solution cen violate the maximum principle, but the amount by which (36) is violated before a violation occurs will surely be problem dependent. Third, the constants, $\xi_{U}, \xi_{L}, \eta_{U}$, $\eta_{L}$, and $\alpha$ are independent of an opacity multiplier, but $\gamma$ is inversely proportional to an opacity multiplier. Therefore, if we introduce an opacity multiplier $\lambda$ :

$$
\sigma(v, T) \rightarrow \lambda \sigma(v, T)
$$

then we must scale $\Delta t$ as

$$
\Delta t \rightarrow \frac{\Delta t}{\lambda}
$$

to guarantee that the maximum principle holds. This implies that as the material becomes optically thick, to satisfy (36) the time steps must be taken smaller.

The Nonlinear Quasi-Implicit (NQI) Method ${ }^{17}$ is a deterministic version of the FC method with a set equal to one. It is described by

$$
\begin{aligned}
& \frac{I_{n+1}-I_{n}}{c \Delta t_{n}}+\underline{\Omega} \cdot \underline{\nabla} I_{n+1}=\sigma\left(\frac{B_{n}}{\phi_{n}} \phi_{n+1}-I_{n+1}\right), \\
& \frac{\phi_{n+1}-\phi_{n}}{\Delta t_{n}}=B_{n} \iint \sigma_{n}\left(I_{n+1}-\frac{B_{n}}{\phi_{n}} \phi_{n+1}\right) d v d \Omega .
\end{aligned}
$$

Let us define 


$$
\begin{aligned}
& \theta_{U}=\sup _{T_{L}<T<T_{U}} \frac{c_{V}(T)}{4 \pi} \iint \sigma(v, T) \frac{T B\left(v, T_{U}\right)-T_{U} B(v, T)}{T_{U}-T} d v d \Omega, \quad \text { (40a) } \\
& \theta_{L}=-\sup _{T_{L}}<\frac{C_{V}(T)}{4 \pi} \iint T_{U} \frac{T_{L} B(\nu, T)-T B\left(\nu, T_{L}\right)}{T-T_{L}} d v d \Omega \cdot(40 b)
\end{aligned}
$$

It can easily be shown that these constants are positive. We then have

Maximum Principle for the NQI Method: If the time step $\Delta t$ satisfies

$\Delta t\left[\max \left(\theta_{U}, \theta_{L}\right)\right]<1$,

then the NQI method satisfies the maximum principle.

The Linear Quasi-Implicit ${ }^{18}$ (LQI) method is a second deterministic method for solving problem (11). It is defined by

$$
\begin{aligned}
& \frac{I_{n+1}-I_{n}}{c \Delta t_{n}}+\underline{\Omega} \cdot \underline{\nabla} I_{n+1}=\sigma_{n}\left[B_{n}+\left(I_{n+1}-T_{n}\right) \frac{\partial B_{n}}{\partial T}-I_{n+1}\right], \\
& c_{v, n} \frac{T_{n+1}-T_{n}}{\Delta t_{n}}=\iint \sigma_{n}\left[I_{n+1}-B_{n}-\left(T_{n+1}-T_{n}\right) \frac{\partial B_{n}}{\partial T}\right] d v d \Omega .
\end{aligned}
$$

For this method, one cannot derive a maximum principle by the type of analysis we used for the FC and NQI methods.

Finally, the Fully Implicit (FI) method is a third determinlstic method for solving Eqs. (11) - (16). Tt is defined by

$$
\begin{aligned}
& \frac{I_{n+1}-I_{n}}{c \Delta t_{n}}+\underline{\Omega} \cdot \underline{\nabla} I_{n+1}=\sigma_{n}\left(B_{n+1}-I_{n+1}\right), \\
& c_{v, n} \frac{T_{n+1}-T_{n}}{\Delta t_{n}}=\iint a_{n}\left(I_{n+1}-B_{n+1}\right) d v d \Omega .
\end{aligned}
$$

Since $B_{n+1}=B\left(v, T_{n+1}\right)$ depends on $T_{n+1}$ nonlinearly, one must solve a nonlinear problem within each time step; this can be accomplished by iteration. Also, note that although the Flanck function is treated implicitly, the opacity and heat capacity are not. A rather detailed analysis yields the following result. 
Existence, Uniqueness, and Maximum Principle for the FI Method: Let the initial and boundary conditions satisfy Eqs. (15). Then for all $\Delta t$, a unlque solution of Eqs. (43) exists, satisfying Eqs. (16).

Remarks: Thus, for the FI method there is no constraint on the time step, but one pays the price in having to iterate to achieve a truly implicit evaluation of the Planckian. Such iteration is straightforward for deterministic metrods, where an iteration process is used anyway (one simply re-evaluates the Planck function within this iteration process), but it does not seem feasible for Morite Carlo methods. Thus, it appears possible, in a practical sense, to implement a deterministic time-differencing method for which the solution obeys the maximum principle for all time steps, but this does not seem possible for Monte Carlo methods.

H. Linking Monte Carlo and $S_{N}$ via Response Matrices ( $W$. L. Fillppone and R. E. Alcouffe)

There have been surprisingly few attempts to develop $\mathrm{S}_{\mathrm{N}}$ /Monte Carlo hybrid techniques. Among the ideas that have been tried are $S_{N}$ adjoint biasing ${ }^{19}$ for Monte Carlo calculations, Monte Carlo-generated first collision sou. $-s^{20}$ for $S_{N}$ calculations, and the random selection 21 of quadrature directions in $S_{N}$ codes. We are now investigating the possibility of combining separate $S_{N}$ and Monte Carlo calculations using response matrix techniques. The hope is to obtain a method suitable for a medium, $W$, consisting of I regions, $V_{i}, W=U_{i=1}^{U} V_{i}$, some of which are optically very thin and others optically very thick. For such a medium, efficient transport solvers are not available. Monte Carlo couputations are extremely time consuming because of the large number of collisions that can occur in the optically thick regijns, whereas $S_{N}$ calculations suffer from the ray effects and large spatial diffurm ?cing errors that can occur in the optically thin regions. In theory the streaming ray (SR) method $^{22,23}$ should be applicable, but an SR production code does not exist and no project to develop such a code is currently being funded. The streaming matrix method 24 could be used, but only if the optically thin regions are simulated by voids.

The Monte Carlo isthod works most efficientiy in optically thin regions, whereas the $S_{N}$ technique with diffusion synthetic acceleration (DSA) performs at its best in optically thick regions. This provides the incentive for 
only in optically thin regions and $S_{N}$ everywhere else. The fluxes at the interface tetween $S_{N}$ and Monte Carlo regions can be determined iteratively. Once these iluxes are known, the interior fluxes are easily determined.

To $1 \mathrm{mplement}$ the method, all interface fluxes must be defined numerlcally on the $S_{N}$ grid. Thus, for any region $V_{1}$ the interface fluxes will be represented by vectors of dimension

$$
K_{1}=C_{i} \cdot G \cdot M / 2 \text {, }
$$

where $C_{i}$ is the total number of $S_{N}$ mesh cells bordering the interface (as opposed to exterior) surfaces of $V_{i}, G$ is the number of energy groups, and $M=N(N+2)$ (in three dimensions) is the total number of discrete directions. The response matrix $R_{1}$ for $V_{1}$ is defined such that

$$
\vec{\phi}_{1}^{+}=R_{i} \vec{\phi}_{1}^{-}+\vec{S}_{i}
$$

where

$$
\begin{aligned}
& \vec{\phi}_{1}= \\
\vec{\phi}_{i}^{+}= & \text {the interface flux of particles entering } v_{i}, \\
\vec{S}_{i}= & \text { the interface flux of particles that would leave region } i \text { under } \\
& \text { vacuum }\left(\vec{\phi}_{1}=0\right) \text { boundary conditions. This flux is due to sources } \\
& \text { within } v_{i} \text { and any boundary sources that may }=x i s t \text { on a common } \\
& \text { surface of } v_{i} \text { and } w .
\end{aligned}
$$

Clearly, a knowledge of the $\vec{\phi}_{i}^{+}$is sufficient to determine the $\vec{\phi}_{i}^{-}$. Therefore, the interface fluxes can be obtained from the simple iteration procedure,

$$
\vec{\phi}_{i}^{+}(P+1)=R_{i} \vec{\phi}_{i}^{-(P)}+\vec{S}_{i}
$$

where the $\vec{\phi}_{i}^{ \pm}(P)$ are the interface fluxes for iteration $P$. The convergence is guaranteed if there are some net losses from the system, and with Monte carlo being used in the optically thin regions good accuracy is anticipated. 
We are interested mainly in problems with conservative scattering and very little leakage. For such problems the convergence of the lnterface flux iterations will be very slow. Therefore, two questions need to be answered before the effectiveness of the method can be evaluated:

(1) Should the response matrices be precalculated?

(2) Can the interface flux iterations be accelerated?

1. Precalculated Response Matrices. The iterations indicated in Eq. (45) can be implemented in two ways. First, if $R_{1}$ is precalculated, $\vec{\phi}_{i}+(P+1)$ is obtained from $\vec{\phi}_{1}^{-(P)}$ and $\vec{S}_{i}$ using a matrix multiplication and a vector addition. Alternatively, with the boundary flux $\vec{\phi}_{1}^{-(P)}$ specified, $\vec{\phi}_{i}^{+(P+1)}$ can be determined by numerically solving the transport equation in $V_{i}$. Our current thinking is to use the first approach if $V_{1}$ is opticr? ly thin (Monte Carlo region) and the alternative approach if $V_{i}$ is optically thick $\left(S_{N}\right.$ region).

Consider first an $\mathrm{S}_{\mathrm{N}}$ region. The response matrix calculation would be $\mathrm{K}_{\mathrm{i}}$ times more costly than a single interface flux calculation. The total number of interface iterations, $P$, would have to be comparable to $K_{i}$ to make precalculation competitive. In addition, storing $R_{i}$ would require $K_{i}^{2}$ locations. We expect (with some type of acceleration) that

$P<K_{i} \quad$

If this is not so, we would probably start looking for a new method of solution. Thus, precalculating the $S_{N}$ response mairices does not appear to be a realistic possibility.

The situation for the optically thin regions is quite different. A Monte Carlo response matrix calculation requires roughly the same computation time as a single boundary value problem. To see why, let us consider the Monte Carlo determination of $\vec{\phi}_{i}^{+}(P+1)$ from $\vec{\phi}_{i}^{-(P)}$ assuming that $\vec{S}_{1}$ has already been determined. We will make use of the following definitions:

$$
\begin{aligned}
S_{i k} & =k^{\text {th }} \text { component of } \vec{S}_{i}, \\
\phi_{1 k}^{ \pm(P)} & =k^{\text {th }} \text { component of } \vec{\phi}_{1}^{ \pm(P)}
\end{aligned}
$$




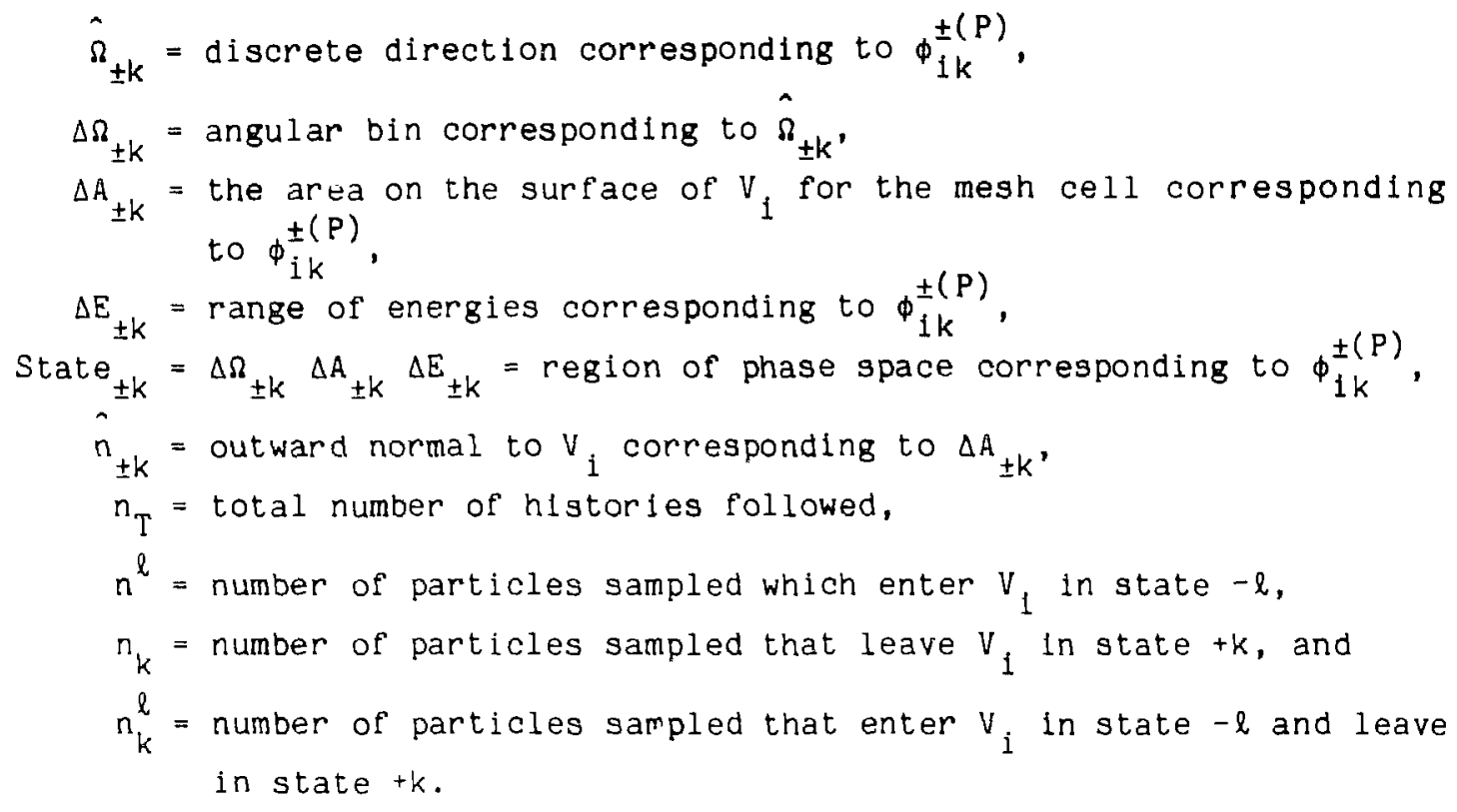

The Monte carlo estimate for $\phi_{i k}^{+(P+1)}$ is

$$
\phi_{i k}^{+(P+1)}=\frac{1}{\Delta A_{k} \hat{\Omega}_{+k} \cdot \hat{n}_{+k}}\left(\frac{n_{k}}{n_{T}}\right) J^{-(P)}+S_{1 k} \text {, }
$$

where $J^{-(P)}$ is the total rate at which particles enter $V_{i}$ during iteration step P. Its numerical definition is

$$
J^{-(P)}=\sum_{k=1}^{K_{i}} \phi_{i k}^{-(P)}\left|\hat{\Omega}_{-k} \cdot \hat{n}_{-k}\right| \Delta A_{-k}
$$

From the definitions of $n_{k}$ and $n_{k}^{l}$,

$$
n_{k}=\sum_{\ell=1}^{k} n_{k}^{\ell}
$$

and with unbiased source sampling,

$$
n^{\ell}=\frac{\phi_{i \ell}^{-(P)}\left|\hat{\Omega}_{-\ell} \cdot \hat{n}_{-\ell}\right| \Delta A_{-\ell}}{J^{-(P)}} n_{T}
$$


Therefore, combining Eqs. (48), (50), and (51) we obtain

$$
\phi_{i k}^{+(F+1)}=\sum_{\ell=1}^{K}\left[R_{i}\right]_{k}^{\ell} \phi_{i \ell}^{-(P)}+i_{i k} .
$$

where

$$
\left[R_{i}\right]_{k}^{\ell}=\frac{\left|\hat{\Omega}_{-\ell} \cdot \hat{n}_{-\ell}\right| \Delta A_{-\ell}}{\Delta A_{k} \hat{\Omega}_{+k} \cdot \hat{n}_{+k}} \frac{n^{\ell}}{n^{\ell}} .
$$

The $\left[R_{i}\right]_{k}^{l}$ are recognized as the matrix elements of $R_{i}$.

To obtain $\vec{\phi}_{1}^{+}(\mathrm{P}+1)$ directly from Eq. (48) requires a total of $\mathrm{n}_{\mathrm{T}}$ histories. However, with these same historles, we can obtain through Eqs. (52) and (53) the identical estimate for $\phi_{1}^{+(P+1)}$ and the $\left[R_{i}\right]_{k}^{l}$ as well. That is, no additional histories are needed to obtain $R_{1}$. The only costs are the $k_{1}^{2}$ storage locations required $f_{\text {or }} R_{i}$ and a somewhat more complicated scoring routine. To determine the $n_{k}^{l}$ it is necessary to consider the entrance and exit state of each particle followed, whereas for $n_{k}$, only the exit state is relevant.

One minor point may need clarification. The $e^{\text {th }}$ column of $R_{1}$ is determined using $n^{\ell}$ histories. As described above, Eq. (51), $n^{\ell}$ depends on $P$, so because of statistical fluctuations, $R_{i}$ will change slightly with each iteration. Obviously, it would be ill advised to calculate a new $R_{1}$ at each iteration step. Our intention is to calculate the $R_{i}$ with $P=0$ in Eq. (51), and then use the same $R_{i}$ until convergence of the interface fluxes is reached. At this point additional histories could be followed, increasing $n_{T}$ and some of the $n^{\ell}$ so as to make the source sampled correspond to $\vec{\phi}_{i}^{-(\infty)}$ rather than $\vec{\phi}_{i}^{-(0)}$. If the $R_{1}$ were significantly altered by the added histories, the interface flux iteration could be repeated. The important point is that no histories need be wasted.

Two Monte Carlo calculations are required for each optically thin region, one to determine $\vec{s}_{1}$ and one (with possibie updates) to determine $R_{1}$. The constraint is that enough memory be available to store the $R_{1}$. Ironically, the size of the Monte Carlo response matrices is determined by the number of $\mathrm{S}_{\mathrm{N}}$ states required on the surfaces of the surrounding optically thick regions. 
2. Accelerating the Interface Flux Iterations. Although DSA is extremely effective for acrelerating the source iterations in optically thick regions, it is not applicable to Eq. (46), and a different method is needed. We have been experimenting with an extrapolation procedure ${ }^{25}$ that appears to work well. We call it the methor: of residual expansion functions (REF). To describe this technique it will be helpful to express the interface flux iterations by a single equation.

$$
\vec{\Phi}^{ \pm(p)}=\left[\begin{array}{l}
\vec{\phi}_{1}^{ \pm}(p) \\
\vec{\phi}_{2}^{ \pm}(p) \\
\vdots \\
\vec{\phi}_{I}^{ \pm}(p)
\end{array}\right],
$$

and

$$
\vec{S}=\left[\begin{array}{c}
\vec{S}_{1} \\
\vec{S}_{2} \\
\vdots \\
\vec{S}_{I}
\end{array}\right],
$$

which are vectors of dimension

$$
K=\sum_{i=1}^{I} K_{i}
$$

Then, from Eq. (46) it is evident that

$$
\vec{\phi}^{+(p+1)}=R^{\prime} \vec{\Phi}^{-(p)}+\vec{S},
$$

where $R^{\prime}$ is a KxK matrix determined by the $R_{i}, 1=1,2, \ldots I$. The explicit dependence of $R^{\prime}$ on the $R_{1}$ (which is problem specific and potentially very complicated) need not be determined. 


$$
\begin{aligned}
& \text { Clearly, } \vec{\Phi}^{-(p)} \text { is just a permutation of } \vec{\phi}^{+(p)} \text { and therefore, } \\
& \vec{\phi}^{+(p+1)}=R \vec{\phi}^{+(p)}+\vec{S},
\end{aligned}
$$

where

$$
R=R^{\prime} P^{T} \text {, }
$$

and $P^{T}$ is the transpose of the permutation matrix that transforms $\vec{\Phi}^{-(p)}$ into $\vec{\phi}^{+(p)}$. From Eq. (58),

$$
\vec{x}^{(p)}=\overrightarrow{R x^{(p-1)}}=R^{p-1} \vec{x}^{(1)}=\sum_{i=1}^{N(p)} \lambda_{i}^{p-1} a_{i} \vec{\psi}_{i},
$$

where

$$
\vec{x}^{(p)}=\vec{\Phi}^{+(p)}-\vec{\Phi}^{+}(p-1),
$$

$\lambda_{i}=$ the eigenvalue of $\mathrm{R}$ with the $i^{\text {th }}$ largest magnitude,

$\vec{\psi}_{k}=$ the eigenvector corresponding to $\lambda_{k}$ '

$N(P)=$ the number of values of $k$ for which $\lambda_{k}^{p-1} a_{k} \vec{\psi}_{k}$ is not negligible compared to $\lambda_{1}^{\mathrm{p}-1} \mathrm{a}_{1} \vec{\psi}_{1}$, and

$a_{k}=$ the $k^{\text {th }}$ coefficient in the expansion

$$
\vec{x}^{(1)}=\sum_{k=1}^{K} a_{k} \psi_{k}
$$

(Here we are assuming for simplicity of notation that $R$ has a complete set of nondegenerate eigenvectors.) We refer to the condition defined by Eq. (60) as $\mathrm{N}^{\text {th }}$ order shape convergence at iteration $\mathrm{p}$.

The converged solution of Eq. (58), $\vec{\Phi}^{+}=\vec{\Phi}^{+\infty}$, satisfies the equation

$$
\vec{\phi}^{+}=R \vec{\phi}^{+}+\vec{S} \text {. }
$$

It is easy to show that 


$$
\vec{\Phi}^{+}=\vec{\Phi}^{+(p)}+\vec{\varepsilon}^{(p)}
$$

where

$$
\vec{E}^{(p)}=\sum_{n=p^{+1}}^{\infty} \vec{x}^{(n)}
$$

Roughty speaking, the REF method is applicable if the $\vec{x}(p)$ shape converges rapidly, or stated more precisely if there exists a p such that

$$
P=p+N(p)
$$

is an acceptable number of iterations. Since

$$
N(p+q) \leq N(p), q=1,2, \cdots,
$$

it follows from Eqs. (60) and (65) that there exist constants $b_{n}$ and $\alpha_{n}$ such that

$$
\begin{aligned}
\vec{\varepsilon}^{(p)} & =\sum_{n=1}^{N(p)} b_{n} \vec{\psi}_{n} \\
& =\sum_{n=1}^{N(p)} \alpha_{n} \vec{x}^{(p+n)} .
\end{aligned}
$$

Then, using Eq. (64) we obtain an estimate for the exact solution,

$$
\vec{\Phi}^{+}=\vec{\Phi}_{T}^{+(P)} \equiv \vec{\Phi}^{+(P)}+\sum_{n=1}^{N(p)} \alpha_{n} \vec{x}^{(p+n)}
$$

Although the $\alpha_{n}$ could be formally expressed in terms of the $\lambda_{i}$, $\vec{\psi}_{i}$, and $a_{i}$, this would be of no practical value because of the difficult eigenvalue problem. Equation (69) is of value however, because the $\alpha_{n}$ can be determined 
directly from the $\vec{x}^{(n)}$. Substituting for $\vec{\Phi}^{+}$in Eq. (63) from Eq. (69), and using the first equality in Eq. (60), it is possible to show ${ }^{25}$ that

$$
\alpha_{n}=\frac{\sum_{\ell=1}^{n} B_{\ell}}{1-\sum_{\ell=1}^{N(p)} B_{\ell}},
$$

where the $B_{\ell}$ are obtained from a least-squares $f$ it to the equation

$$
\vec{x}^{(P)}=\sum_{n=1}^{N(p)} \beta_{n} \vec{x}^{(n+p-1)} .
$$

Exact equality in Eq. (71) is not possible without exact equality in Eq. (60). The error

$$
\vec{\varepsilon}_{x}^{(p)}=\vec{x}^{(P)}-\sum_{n=1}^{N(p)} \beta_{n} \vec{x}^{(n+p-1)}
$$

is a measure of the error in Eq. (60) and also the error in Eq. (69),

$$
\vec{\varepsilon}_{T}^{(P)} \equiv \vec{\Phi}^{+}-\vec{\Phi}_{T}^{+}
$$

It is possible to show ${ }^{25}$ that

$$
\vec{\varepsilon}_{T}^{(P)}=\left(1+\alpha_{N(p)}\right) R(1-R)^{-1} \underset{\varepsilon_{X}}{\rightarrow}(p)
$$

The REF acceleration scheme can be implemented by the following steps:

(1) Choose a value of $P$.

(2) Determine the $\vec{x}^{(n)}, n=1,2, \ldots$. This is done using Eqs. (46), (54), and (61) rather than Eqs. (58) and (61) so R need not be determined. 
(3) Check for $\mathrm{N}^{\text {th }}$ order shape convergence at $\mathrm{p}=\mathrm{P}-\mathrm{N}(\mathrm{p})$ for $\mathrm{N}=1,2, \ldots$ by computing $\vec{E}_{x}(p)$ and select the value of $p$ that minimizes $\left(1+\alpha_{N(p)}\right) \vec{E}_{X}^{(p)}$, (using Eq. (72) for $\vec{\varepsilon}_{x}^{(p)}$ and $E q .(70)$ for $\left.\alpha_{N(p)}\right)$.

(4) Estimate $\stackrel{+}{\varphi}^{+}$from Eq. (69).

(5) Carry out an additional iteration to see if $\vec{\phi}_{T}^{+}$is an acceptable estimate of $\vec{\phi}^{+}$.

3. Sample Calculations. We have tested the REF acceleration scheme on one-dimensional slab problems and are in the process of testing the entire Monte Carlo/ $\mathrm{S}_{\mathrm{N}}$ ' response matrix method in $(\mathrm{x}, \mathrm{y})$ geometry.

For the one-dimensional calculations we used two $S_{N}$ regions $(I=2)$ and 12 uniformly spaced cosine ordinates $(N=M=12)$. Thus, the interface flux vectors were of dimension $k=k_{1}=K_{2}=M / 2=6$. Table II shows $\vec{x}^{(p)}$ and $\underset{x}{(p)} /|\vec{x}(p)|$ for two different problem configurations. When $V_{1}$ has a thickness of $4 \mathrm{mfp}$, first-order shape convergence is reached, to three significant figures, by Iteration 2. With $v_{1}=12 \mathrm{mfp}$. first-order shape convergence is reached even sooner: to two significant figures by iteration 1 and to four-place accuracy by 1 teration 2 .

Table III shows the errors in the interface flux vectors as determined with and without the REF extrapolation procedure. It is seen that the REF techniques give a dramatic reduction in errors.

Our two-dimensional calculations have involved an optically thin Monte Carlo slab embedded between two optically thick $S_{N}$ regions. The $S_{N}$ calculations were done using the TWODANT code. ${ }^{2}$ A new program was written to calculate the Monte Carlo response matrix for the center region and to implement the REF acceleration. Although the combined algorithm is probably not completely debugged, it does appear that the interface fluxes quickly shape-converge (although somewhat less rapidly than in the one-dimensional case) and the REF method accurately estimates the converged solution.

Comparisons with pure Monte Carlo and pure $S_{N}$ will soon be avallable so the efficiency of the hybrid method can be assessed.

\section{MONTE CARLO RADIATION TRANSPORT}

The Monte Carlo radiation transport effort in Group $x-6$ is devoted to the development, impiementation, assessment, and application of Monte Carlo methods and computer codes for radiation transport. 
TABLE II

INTERFACE RESIDUALS FOR ONE-DIMENSIONAL SLAB CALCULATIONS

\begin{tabular}{|l|l|l|l|l|l|}
\hline$\vec{x}^{(1)}$ & $\vec{x}^{(2)}$ & $\frac{\vec{x}^{(1)}}{\left|\vec{x}^{(1)}\right|}$ & $\frac{\vec{x}^{(p)}}{\left|\vec{x}^{(p)}\right|, P \geq 1}$ & \multicolumn{2}{|c|}{$\begin{array}{l}\text { Slab Th1ckness (mfp) } \\
V_{1}\end{array}$} \\
\hline .1903 & .1295 & .1606 & .1597 & & \\
.1931 & .1316 & .1630 & .1623 & & \\
.1959 & .1337 & .1654 & .1649 & 4 & \\
.1988 & .1359 & .1678 & .1676 & & \\
.2018 & .1384 & .1703 & .171 & & \\
.2049 & .1417 & .1730 & .1747 & & \\
\hline & & .1627 & & \\
.2376 & .1927 & .1612 & .1604 & & \\
.2409 & .1956 & .1634 & .1628 & & \\
.2442 & .1985 & .1657 & .1652 & 12 & \\
.2475 & .2016 & .1679 & .1677 & & \\
.2506 & .2048 & .1700 & .1704 & & \\
.2533 & .2085 & .1718 & .1735 & & \\
\hline
\end{tabular}

TABLE III

PERCENT ERROR IN THE INTERFACE FLUXES WITH AND WITHOUT REF ACCELERATION FOR TWO SLAB PROBLEMS

\begin{tabular}{|c|c|c|c|c|c|c|c|}
\hline \multirow{2}{*}{$\begin{array}{c}\text { SLAB } \\
\text { THICKNESSES } \\
\text { (mfp) } \\
\end{array}$} & \multicolumn{7}{|c|}{ PERCENT ERROR IN INTERFACE FLUXES } \\
\hline & \multicolumn{4}{|c|}{ UNACCELERATED } & \multicolumn{3}{|c|}{ WITH REF ACCELERATION } \\
\hline$v_{1}, v_{2}$ & 1 & 2 & 3 & 4 & 1 & 2 & 3 \\
\hline 4,12 & 49 & 33 & 23 & - & $9 \times 10^{-4}$ & $4 \times 10^{-6}$ & - \\
\hline 12,12 & 64 & 52 & 42 & 34 & $6 \times 10^{-7}$ & $6 \times 10^{-10}$ & $1 \times 10^{-12}$ \\
\hline
\end{tabular}




\section{A. MCNP Support (J. Briesmeister)}

Most of my time this reporting perlod was devoted to the continuing effort of developing a new manual for Version 3 of our MCNP continuous energy Monte Carlo code. ${ }^{26}$ Several appendices were completed and a rough draft of Chapter 3 of the manual was finished and distributed to selected users for comment.

Some time was devoted to consultation with users about MCNP at the National Magnetic Fusion Energy Computing Center (NMFECC) at Livermore. Certain problems with Los Alamos computer codes at the NMFECC are not yet resolved. Version $3 A$ of MCNP and its associated flles were shipped to the NMFECC for trial use in a user-friendly status.

B. Biasing Through a Cylindrical Window from a Spherlcally Symmetric Surface Source in MCNP3A (E. C. Snow)

In many problems where a spherically symmetric surtace source can be used, the variance of the resulting transport calculation can be reduced by biasing the particle trajectories at the source in a direction of interest. In some such cases, this direction can be best defined as a cylindrical window in space through which the particles are required to pass. In the following discussion, a method for biasing the starting trajectories of the source particles in the direction of a cylindrical window is developed, as are the weight reductions required to unbias the results.

The cylindrical window need not be a part of the geometry or even exist at all except as an imaginary window in space because the window is used only to direct the particles as they leave the source. The oniy restrictions on the window are that it be defined such that the symmetric axis of the cylinder pass through the center of the source sphere, that both ends of the window be on the same side of the source sphere, and that the near end of the window not intersect the source sphere. To get the proper location and orientation of the source sphere and cylindrical window, one must first consider the combination as being defined with the source sphere centered at the origin and the window centered on the positive z-axis. One must then develop a TRn card ${ }^{26}$ to translate and rotate this combination properly in the problem geometry.

If the information stored on a surface source is spherically symmetric, the only spatial information of value--in fact, the only spatial information stored in MCNP3A--is the polar angle relative to the surface normal. Given this polar angle, within limits dictated by the window, one can place the 
particle on the source sphere such that, with proper selection of the azimuthal angle, the particle will be started in a direction that will allow it to pass through the window if unobstructed in its flight. In the following discussion, the method for selecting the starting position and associated weight reduction will. be developed. Next, the method for the proper selection of the azimuthal angle and assoclated weight reduction will be developed. Last, the aiming point for the particle trajectory is determined.

The initial parameters that are given are defined as

$$
\begin{aligned}
& R_{s}=\text { source sphere radius, } \\
& R_{p}=\text { window cylinder radius, } \\
& z_{b}=\text { distance to near end of window, and } \\
& z_{d}=\text { distance to far end of window, }
\end{aligned}
$$

as shown in Fig. 11. First, we need to determine the field of view ( $R$ ) of the window at the source by considering the triangle $\mathrm{ABC}$ in Fig. 11.

$$
R_{v s}=\frac{\left(z_{d}+z_{b}\right)}{2} \cdot \sin \alpha_{w} \text {, }
$$

but

$$
\sin a_{w}=\frac{2 R_{p}}{\left[\left(z_{d}-z_{b}\right)^{2}+4 R_{p}^{2}\right]^{\frac{1}{2}}} \text {, }
$$

or

$$
R_{v s}=\frac{\left(z_{d}+z_{b}\right) R_{p}}{\left.\left[\left(z_{d}-z_{b}\right)\right]^{2}+4 R_{p}\right]^{\frac{1}{2}}}
$$

therefore,

$$
R_{v s}=\frac{R_{p}\left(z_{d}+z_{b}\right)}{\left[\left(z_{d}-z_{b}\right)^{2}+4 R_{p}^{2}\right]^{\frac{1}{2}}}
$$

We can see from the figure that if $R_{v s} \geq R_{s}$ or $R_{p} \geq R_{s}$, then $\theta_{m}=90^{\circ}$, and there is no restriction on the polar angle of the particle trajectory 48 


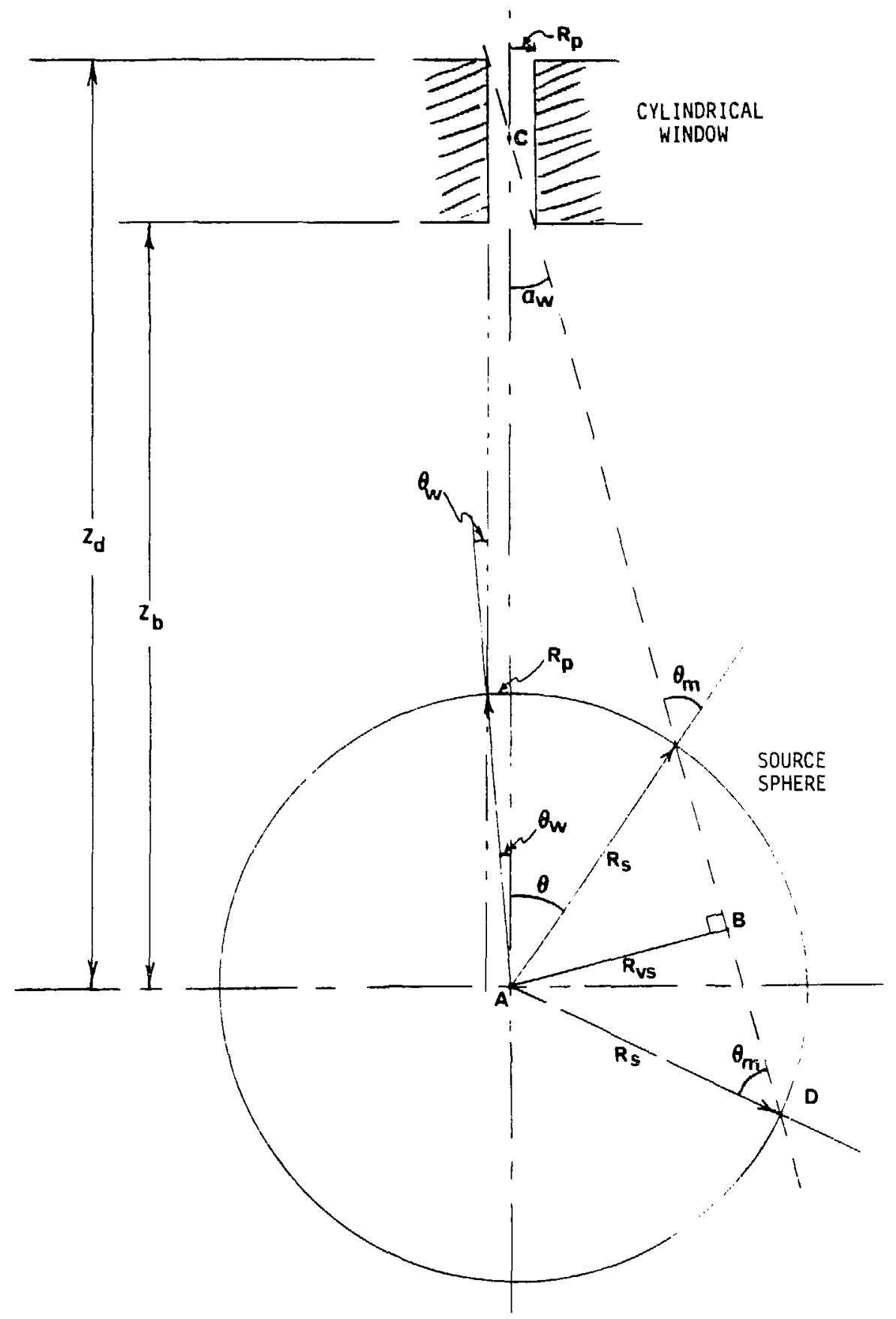

Fig. 11. Model fur biasing through a cylindrical window from a spherically symmetric surface source. 
relative to the surface normal. This means that all particles can be directed through the window.

If $R_{v s}<R_{s}$, as shown in Fig. 11, we must determine the maximum acceptable polar angle $\left(\theta_{m}\right)$ by considering the $r i g h t$ triangle ABD, as shown in this figure. We see that

$$
\theta_{m}=\arcsin \frac{R_{v s}}{R_{s}} \text {. }
$$

Another angle of interest is the angle between the surface normal and the projection of the window cylinder at the source sphere, $\theta_{w}$ in Eig. 11.

$$
\theta_{w}=\arcsin \frac{R_{p}}{R_{s}} .
$$

Next, we need to consider the area on the source sphere where the particle may be placed to ensure its eventual passage through the window. This area, shown as $\Delta A$ ir Fig. 12, is determined by the angles $\theta_{i}$ and $\theta_{0}$ and depends on $\theta_{p}$, the polar angle of the particle trajectory relative to the surface normal. First, we need to determine some constants, shown in $F i g .13$ as $Y_{i}$, $Y_{0}, R_{i}$, and $R V_{0}$.

$$
\begin{aligned}
& Y_{i}=\arctan \frac{R_{p}}{Z_{d}}, \\
& Y_{0}=\arctan \frac{R_{p}}{Z_{b}}, \\
& R V_{i}=\left[Z_{d}^{2}+R_{p}^{2}\right]^{\frac{1}{2}}, \text { and } \\
& R V_{0}=\left[z_{b}^{2}+F_{p}^{2}\right]^{\frac{1}{2}} .
\end{aligned}
$$

When $\theta_{p}>\theta_{w}$, the particle can start outside the area that represents the projection of the window cylinder on the source sphere. In this case, shown in Fig. 13, the inner angle limit $\theta_{i}$ is determined at the far end of the window. and the outer angle limit $\theta_{0}$ is determined at the near end of the window. We can see that 


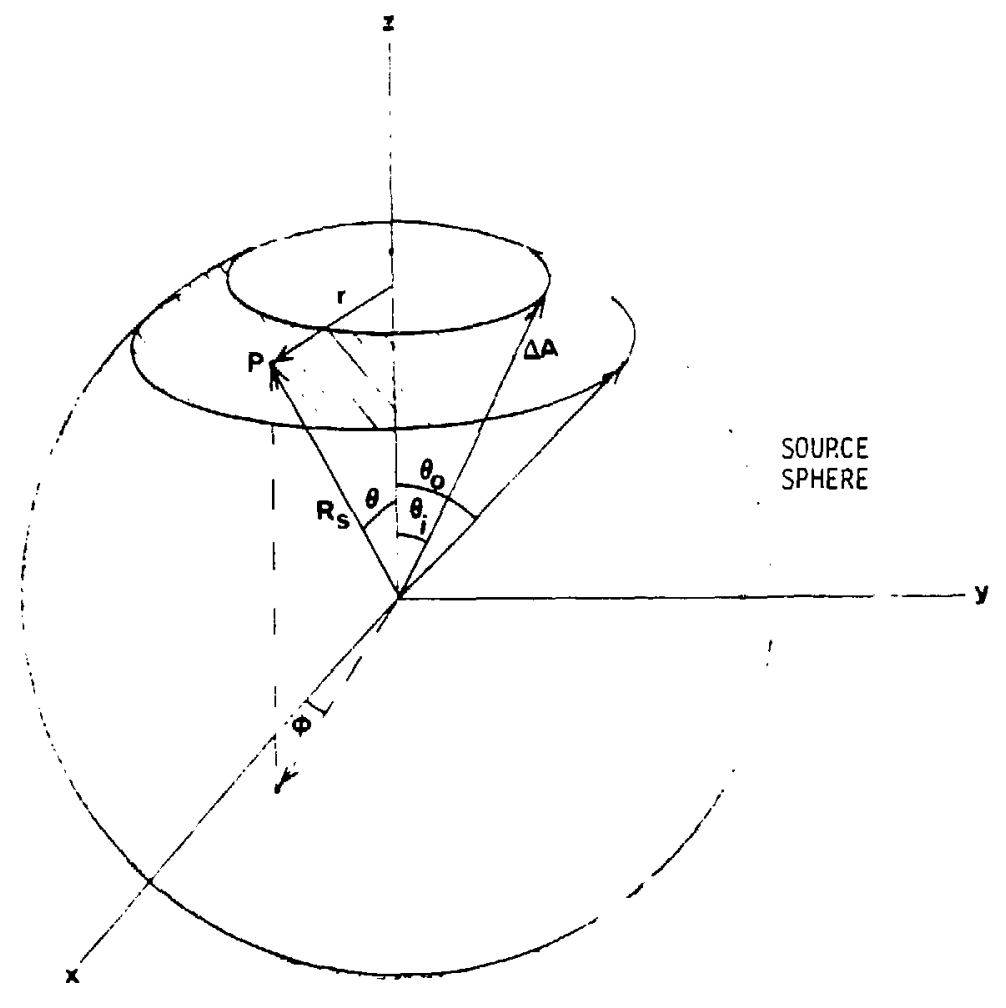

Fig. 12. Area, $\Delta A$, on the source sphere where particle may be placed to ensure passage through cylindrical window. 


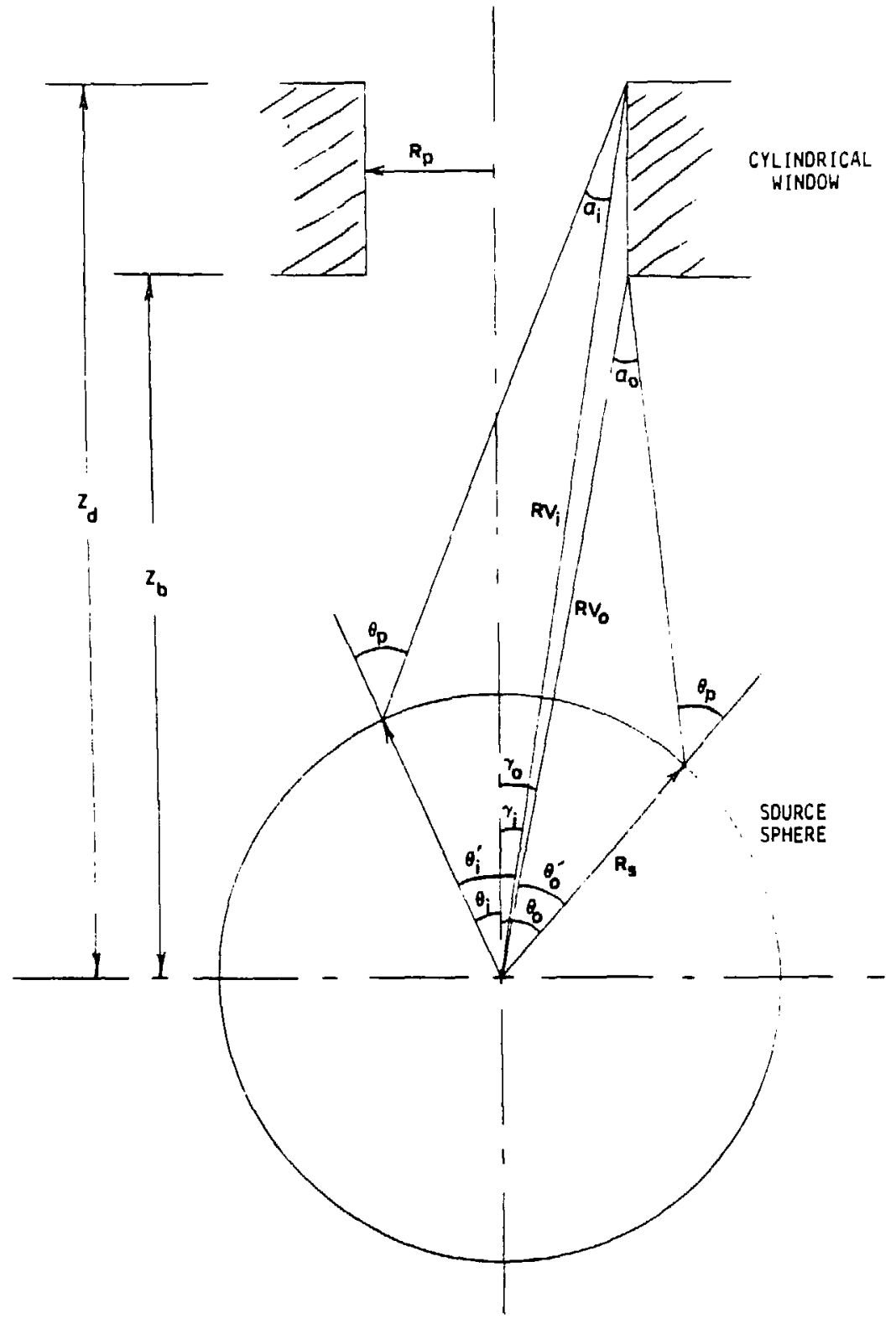

Fig. 13. Illustration showing the quantities $\gamma_{1}, \gamma_{0}, R V_{1}, R_{0}$ and $O_{0}$ when $O_{p}>O_{w}$. 


$$
\theta_{i}=\theta_{i}-Y_{i},
$$

and

$$
\theta_{1}^{\prime}=180^{\circ}-\alpha_{1}-\left(180^{\circ}-\theta_{p}\right),
$$

or

$$
\theta_{i}=\theta_{p}-\gamma_{i}-\alpha_{i}
$$

but

$$
\alpha_{i}=\arcsin \left\{\frac{R_{s}}{R V_{i}} \cdot \sin \left(180^{\circ}-\theta_{p}\right)\right\} ;
$$

therefore, since $\sin \left(180^{\circ}-x\right)=\sin x$,

$$
\theta_{1}=\theta_{p}-r_{i}-\arcsin \left\{\frac{R_{s}}{R V_{i}} \cdot \sin \theta_{p}\right\rfloor .
$$

Erom the same figure,

$$
\theta_{0}=\theta_{p}+\gamma_{0}-\arcsin \left\{\frac{R_{S}}{R V_{0}} \cdot \sin \theta_{p} \downarrow .\right.
$$

When $\theta_{p} \leq \theta_{w}$, the particle can "see" the entire far end of the window, and the near end is not a limiting factor. We then consider the angle $\gamma_{w}$ as shown in Fig. 14 and given by

$$
\gamma_{W}=\arctan \frac{R_{p}}{Z_{d}-R_{s}} .
$$

If $\theta_{p} \leq Y_{w}$, then the inner angle limit goes to zero, or $\theta_{i}=0$. If $\gamma_{w}<\theta_{p} \leq$ $\theta_{w}, \theta_{i}$ is determined as shown above. Then, however, $\theta_{0}$ is determined by the far end of the window, as shown in Fig. 14. We see that

$$
\theta_{0}=\theta_{0}^{\prime}+Y_{1}
$$




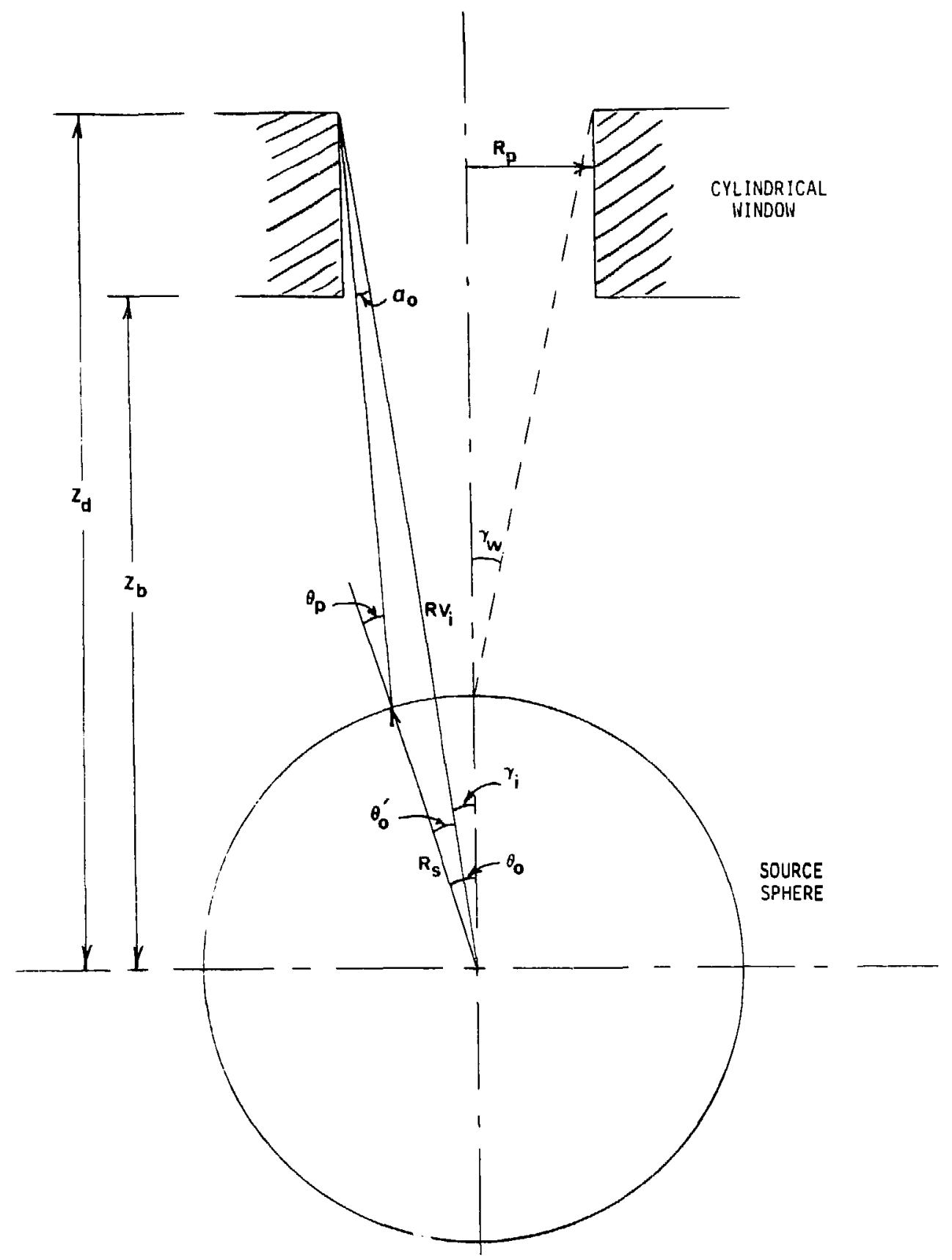

Fig. 14. Illustration of angles $\gamma_{w}$ and $\theta_{0}$ for the case $\theta_{p} \leq \theta_{w}$.

54 
or

$$
\theta_{0}=\theta_{p}+Y_{1}-\alpha_{0} ;
$$

therefore,

$$
\theta_{0}=\theta_{p}+\gamma_{i}-\arcsin \left\{\frac{R_{s}}{R V_{i}}-\sin \theta_{p}\right\} .
$$

For the welght reduction required because we are restricting the area of the sphere in which the particle will be started, we again consider $\Delta A$ in Fig. 12, and we can see that

$$
W t^{\prime}=W t \cdot \frac{\Delta A}{A},
$$

Where 'Wt' is the reduced weight, Wt is the particle welght that was recorded on the surface file, $A$ is the total surface area of the source sphere, and $\Delta A$ is the area in which the particle will be started as described earlier. First, consider determining $\Delta \mathrm{A}$, using

$$
d A=2 \pi r \cdot R_{S} d \theta
$$

where

$$
r=R_{s} \sin \theta \text {, }
$$

so

$$
d A=2 \pi R_{S}^{2} \sin \theta d \theta \text {, }
$$

then

$$
\Delta A=2 \pi R_{s}^{2} \int_{\theta_{i}}^{\theta} \sin \theta d \theta
$$

or 


$$
\begin{aligned}
& \Delta A=2 \pi R_{S}^{2}\left(\cos \theta_{i}-\cos \theta_{0}\right) \\
& \text { If we consider tho area of the sphere, A is given by } \\
& A=4 \pi R_{S}^{2},
\end{aligned}
$$

and

$$
\frac{\Delta \mathrm{A}}{\mathrm{A}}=\frac{1}{2}\left(\cos \theta_{i}-\cos \theta_{0}\right)
$$

therefore,

$$
W^{\prime}=W t \cdot \frac{1}{2}\left(\cos \theta_{i}-\cos \theta_{0}\right) \text {. }
$$

To place the particle randomly in the area $\Delta A$ on the source sphere, we need to sample $\cos \theta$ uniformly between $\cos \theta_{i}$ and $\cos \theta_{0}$ and the azimuthal angle $\$$ uniformly between $0^{\circ}$ and $360^{\circ}$. For $\theta$, we use

$$
\cos \theta=\cos \theta_{i}-\xi_{1}\left(\cos \theta_{i}-\cos \theta_{0}\right)
$$

where $\xi_{n}$ is a random number between 0 and 1 . Therefore,

$$
\theta=\operatorname{arcos}\left\{\cos \theta_{i}-\xi_{1}\left(\cos \theta_{i}-\cos \theta_{0}\right)\right\} \text {, }
$$

and

$$
\phi=\xi_{2} \cdot 360^{\circ} .
$$

From these two angles in polar coordinates and the radius of the source sphert, the coordinates of the point on the source sphere where the particle will be st.arted can be determined from

$$
\begin{aligned}
& X=R_{S} \sin \theta \cos \phi \\
& Y=R_{S} \sin \theta \sin \phi
\end{aligned}
$$




$$
Z=R_{s} \cos \theta
$$

Next, we need to determine the limits of the azimuthal angle of the particle trajectory such that it will be started toward the window. If the selected angle $\theta$ is less than or equal to $\theta_{w}$, the particle will be placed inside the projection of the window on the source sphere. In this area, the particle can "see" the entire far end of the window, and the near end need not be considered when determining the azimuthal angle limits. If $\theta$ is greater than $\theta_{w}$, both ends of the window will have to be considered, with the more restrictive one being used in the final determination of the limits.

To determine the azimuthal angle of acceptance, $\alpha_{m}$, consider the drawing in Fig. 15. We have selected the point $P$ such that $\theta$ is between $\theta_{i}$ and $\theta_{0}$, as dictated by $\theta_{p}$, the particle polar angle relative to the radial vector at the source surface. First, we define a window sphere at radius RV that contains the window circle of the end of the window we are considering. If we now consider all possible paths for a particle with surface polar angle $\theta_{p}$ and starting from point P, they will "sweep" out a cone in space, the axis of which will be a radial vector of the source sphere. The intersection of this cone with the sphere at RV will also be a circle, indicated as the particle path circle in Fig. 15. This circle will be perpendicular to the radial vector at point $P$ at a distance " $d "$ from the center of the source sphere and intersecting the window circle at points $A$ and $B$. The position on the arc of the particle path circle between $A$ and $B$, picked uniformly and at random, is where the particle trajectory must be aimed to pass through this end of the window. If both ends of the cylindrical window are involved, the particle must be aimed so as to pass through both window circles. Therefore, the more restrictive one is used. First, the value of " $d$ " and $R_{c}$, the radius of the particle path circle, must be determined. From Fig. 15,

$$
d=R V \cdot \cos \eta,
$$

but

$$
\eta=180^{\circ}-\eta^{\prime}-\left(180^{\circ}-\theta_{p}\right)
$$




$$
\eta=\theta_{p}-\eta^{\prime}
$$

with

$$
n^{\prime}=\arcsin \left(\frac{R_{s}}{R V} \cdot \sin \theta_{p}\right)
$$

then

$$
n=\theta_{p}-\arcsin \left(\frac{R_{s}}{R V} \cdot \sin \theta_{p}\right)
$$

therefore,

$$
d=R V \cdot \cos \left\{\theta_{p}-\arcsin \left(\frac{R s}{R V} \cdot \sin \theta_{p}\right)\right\} .
$$

We also see that

$$
R_{C}=R V \cdot \sin \eta
$$

or

$$
R_{C}=R V \cdot \sin \left\{\theta_{p}-\arcsin \left(\frac{R_{s}}{R V} \cdot \sin \theta_{p}\right)\right\} .
$$

If we now consider the line between $A$ and $B$ in Fig. 15, we see that it is a common chord of both the window circle and the particle path circle. We can also see that, as chords of these circles, this line is perpendicular to both $a_{p}$ and $a_{c}$ and, therefore, the figure formed by $a_{p}, a_{c}, d$, and $z$ lies in $a$ plane, as shown in Fig. 16. If we can determine $a_{c}$, the distance to the chord $A B$, we can determine the azimuthal angle limit $\alpha_{m^{*}}$ Consider

$$
\ell=\frac{\mathrm{d}}{\cos \gamma} \text { and } \ell=\frac{\mathrm{z}}{\cos \tau}
$$

Also,

$$
\tau=\theta-Y,
$$




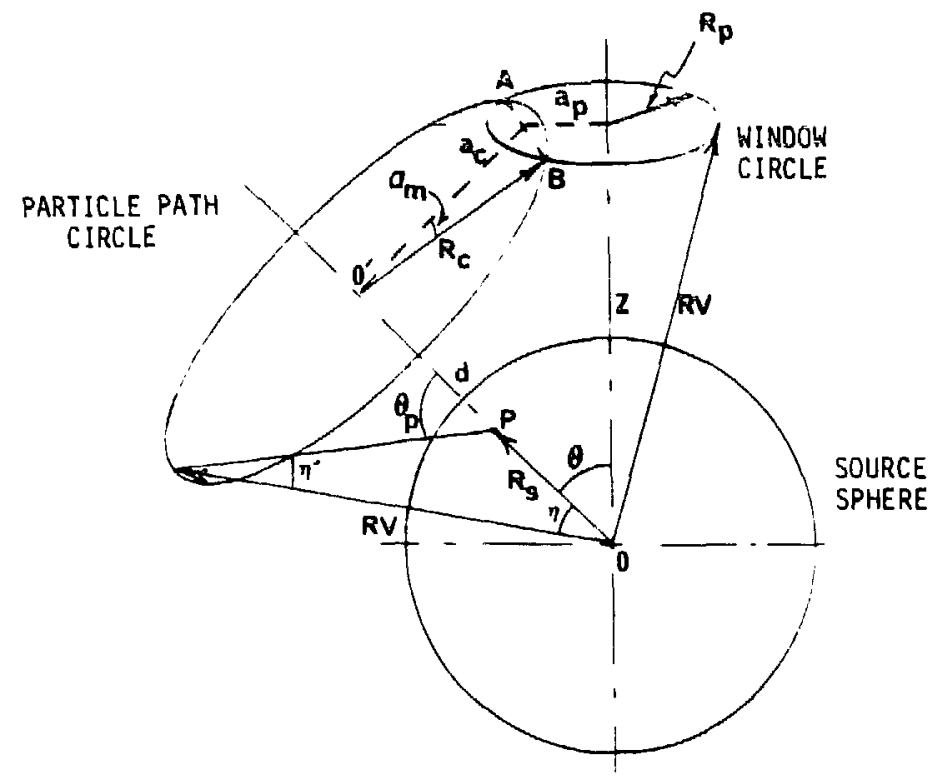

Fig. 15. Illustration showing the angle of acceptance, $\alpha_{m}$.



Fig. 16. The plane formed by the lines $a_{p}, a_{c}, d$, and $z$ from Fig. 15. 
and

$$
\frac{d}{\cos \gamma}=\frac{z}{\cos (\theta-y)}
$$

Using

$$
\cos (\theta-\gamma)=\cos \theta \cos \gamma+\sin \theta \sin \gamma,
$$

and rearranging

$$
\frac{\cos \theta \cos \gamma+\sin \theta \sin \gamma}{\cos \gamma}=\frac{z}{d} \text {, }
$$

or

$$
\tan \gamma=\frac{1}{\sin \theta}\left(\frac{z}{d}-\cos \theta\right),
$$

but

$$
a_{c}=d \tan \gamma:
$$

therefore,

$$
\alpha_{m}=\operatorname{arcos}\left\{\frac{d}{R_{c} \cdot \sin \theta} \cdot\left(\frac{z}{d}-\cos \theta\right)\right\},
$$

where $\pm \alpha_{m}$ are the acceptance angle limits for the azimuthal angle of the particle trajectory.

When $\theta_{p}$ is less than $\gamma_{w}$ (see Fig. 14), the particle path circle is smaller than the window circle and can fall completely inside it, in which case the azimuthal angle is not limited. This occurs when $\eta+\theta$, as shown in Fig. 15, is less than $\gamma_{i}$, as shown in Fig. 13. If, however, $\pi+\theta$ is greater than $\gamma_{i}$, we would use the same expression as above to determine $\alpha_{m}$. Note that since "d" is now greater than " $z, " z / d<1$, and that

$$
-R_{c} \leq a_{c} \leq R_{c}
$$

and that 


$$
0 \leq \alpha_{\mathrm{m}}<\pi
$$

To select the azlmuthal angle of the particle trajectory, we sample the angle $\alpha$ uniformly between the limits $\pm \alpha_{m}$,

$$
\alpha=2 \xi \alpha_{m}-\alpha_{m}
$$

or

$$
\alpha=(2 \xi-1) a_{m},
$$

where $\xi$ is a random number between 0 and 1 .

Because we did not sample the total azimuthal extent of $360^{\circ}$, the weight must be reduced again to unbias the results.

$$
w t^{\prime \prime}=w t^{\prime} \cdot \frac{2 a_{m}}{360^{\circ}}
$$

or

$$
w t^{\prime \prime}=w t^{\prime} \cdot \frac{\alpha_{m}}{180^{0}}
$$

where Wt' is the reduced weight requirod when the particle position on the source sphere was selected.

To locate the final aiming point on the particle path circle, given as $\left(X_{f}, Y_{f}, Z_{f}\right)$ in Fig. 17, first we locate the point on the $z$-axis where the extension of $a_{c}$ would intersect.

$$
z^{\prime \prime}=\frac{d}{\cos \theta}
$$

where " $\mathrm{d}$ " and $\theta$ are as shown in Figs. 15, 16, and 17. We can now determine the direction cosines along $a_{c}$ as

$$
\begin{aligned}
& U^{\prime}=\frac{-x}{s}, \\
& V^{\prime}=\frac{-y}{s},
\end{aligned}
$$




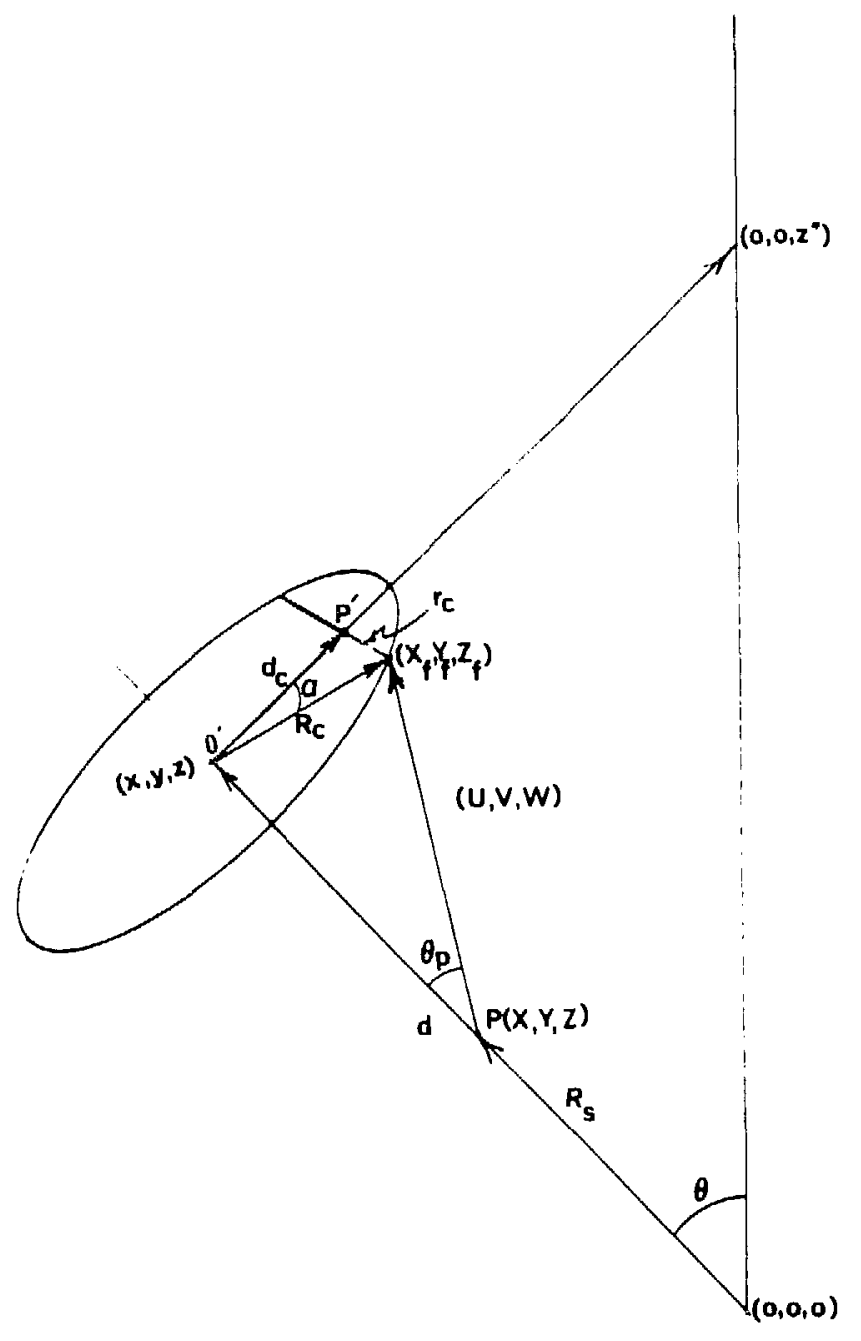

Fig. 17. Final aiming point $\left(\mathrm{X}_{\mathrm{f}}, \mathrm{Y}_{\mathrm{f}}, \mathrm{Z}_{\mathrm{f}}\right)$ on the particle path circle. 


$$
W^{\prime}=\frac{\left(z^{\prime \prime}-z\right)}{s}
$$

where

$$
S=\left[x^{2}+y^{2}+\left(z^{\prime \prime}-z\right)^{2}\right]^{\frac{1}{2}}
$$

or

$$
S=d \tan \theta
$$

The coordinates of $P^{\prime}$ can be found by moving a distance $d_{c}$ along this direction from the point $0^{\prime}$, where

$$
d_{c}=R_{c} \cos \alpha,
$$

and

$$
r_{C}=R_{C} \sin \alpha ;
$$

therefore,

$$
\begin{aligned}
& X^{\prime}=x+d_{c} U^{\prime}, \\
& Y^{\prime}=y+d_{c} V^{\prime}, \text { and } \\
& Z^{\prime}=z+d_{c} W^{\prime},
\end{aligned}
$$

The final point on the arc of the particle path circle is found by moving a distance $r_{c}$ along the chord at $d_{c}$ from the center. Recall that the azimuthai angle $\phi$ that was selected to locate the point $P$ on the source sphere is just the angle we need to calculate the $\Delta x$ and $\Delta y$ from point $P^{\prime}$. Using the fact that this chord at $d_{c}$ of length $r_{c}$ is parallel to the $x-y$ piane,

$$
\begin{aligned}
& X_{f}=X^{\prime}-r_{c} \sin \phi, \\
& Y_{f^{\prime}}=Y^{\prime}+r_{c} \cos \phi,
\end{aligned}
$$




$$
z_{f}=Z^{\prime}
$$

Knowing that the particle must leave $(X, Y, Z)$ and start in the direction of $\left(x_{f}, y_{f}, Z_{f}\right)$, we can now determine the direction cosines required for transport. These are given as

$$
\begin{aligned}
& U=\frac{X_{f}-X}{S^{\prime}}, \\
& V=\frac{Y_{f}-Y}{S^{\prime}}, \text { and } \\
& W=\frac{Z_{f}-Z}{S^{\prime}},
\end{aligned}
$$

where

$$
S^{\prime}=\left[\left(x_{f}-x\right)^{2}+\left(y_{f}-y\right)^{2}+\left(z_{f}-z\right)^{2}\right]^{\frac{1}{2}}
$$

We now have the required values of $X, Y, Z$ and $U, V, W$ to ensure that the particle will start toward the window and that the polar angle of the particle trajectory with respect to the surface normal has been preserved.

\section{Elimination of the Law 18 Sampling Scheme from MCNP (H. M. Fisher)}

Considerable progress has been made toward replacing Law 18 in the MCNP code. $^{26}$ It had been proposed by Bob Little 27 that Law 18 , which involves the use of hard-wired data in Subroutine IFTSP (determines if the energy of a neutron emitted from a fission event is to be sampled from a fission spectrum or an evaporation spectrum) and function FISP (performs the actual sampling of th energy) be replaced by a combination of Law 7 (Simple Fission Spectrum) and Law 9 (Neutron Evaporation Spectrum).

A patch has been written (hopefully to be implemented when Version $3 \mathrm{~A}$ of MCNP is made public) to do this. The patch and the respective CDC-7600 data files for those nuclides that use Law 18 are available on CFS as /098434/LSCAII/ and 1098434/LSCANOUT/FILENAME, respectively, for Los Alamos users.

LSCAN1 scans each nuclide in a given cross-section file to determine if Law 18 has been used. Whenever use of Law 18 has been acknowledged, it is 
replaced by a combination of Law 7 and Law 9. The basic format of the crosssection flles remains the same; however, the data tables have been modifled to reflect the fact that Law 18 and the hard-wired energy-dependent temperature data in the subroutine FISP have been replaced.

D. HETC and Related Improvements (R. E. Prael)

1. New Tally Option for HTAPE. A new tally option has been written for HTAPE, the output analysis code for HETC. 28 In the new scheme, any particle exiting the system scores into a bin determined by the polar and azimuthal angles of 1 ts direction vector with resfect to any combination of the Laboratory coordinates. The effect is equivalent to calculating the current across a sphere with radius much greater than the dimensions of the interactive region located at the origin; the binning by polar and azimuthal angles then corresponds to binning the current by segments defined by latitude and longitude on the sphere. It may also be interpreted as estimating the integral of an angular flux over an element of solid angle at a gredt distance in a void from the interacting region.

2. The HETC Code System at MFE. In response of a number of requests, the entire HETC code system ${ }^{28}$ has been installed at the MFE computing center. During that effort, the LANL versions of CFT, LDR, and BUILD from machine $V$ were moved to NMFECC to permit complete simulation of the Los Alamos environment for generation of executable code. Compilation, loading, and execution of the complete system has been achieved on machine $D$ at NMFECC.

3. Antiproton Annihilation Source for HETC. A special version of HETC, created at the request of Mike Hynes $(P-15)$, features a source routine to read a file containing multiple pion source data from antiproton-proton annihilation events. Each record in the file represrnts the start of one history from an annihilation event and contains the kinematic parameters for two to eight pions (+, -, or 0$)$. The source input file is generated by the NVRTEX code of Mike Hynes.

The new code capability is intended for studying the containment of the energy produced by antiproton annihilation. The charged pions transport through material, creating secondary particles and decaying into muons. The neutral pions decay immediately to high-energy gamma rays, the transport of which will be computed with the TIGER series codes. 29

4. Fermi Breakup Model for HETC. The INCA2 code describes the breakup of an excited nucleus using the Fermi breakup model rather than the evaporation 
model currently used in HETC. It has significant advantages for the description of the de-excitation process in light nuclei. 30 The objective of recent work has been to reduce the INCA2 code to a subroutine package for implementation into HETC as a replacement for the evaporation model for nuclei with $A<18$. The principal simplification that has been made is to limit the allowed breakup modes to two- and three-body channels only, permitting the precalculation of all the branching ratios for the breakup of known nuclear levels with a modest storage requirement. In addition, the logic has been modified to permit the precalculation of ail the energy-independent factors for the breakup probabilities of allowed initial states arising from the intranuclear cascade.

The new code has been tested as a stand-alone code. Implementation into HETC will occur in the near future.

\section{E. Ring Tally for CYLTRAN (H. G. Hughes)}

The Los Alamos version of CYLTRAN, ${ }^{31}$ the cylindrical-geometry member of the Integrated TIGER Series of Monte Carlo electron/photon transport codes, 29 now includes the option of subdividing the transmission and reflection surfaces into radial bins for tallying purposes. The set of all geometric zones in a CYLTRAN problem must comprise a right circular cylinder called the "problem cylinder," with axis along the z-axis of the coordinate system. The circular face of the problem cylinder having maximum $z$-value is called the trarsmission surface; the face with minimum $z$-value is the reflection surface. Tre new option allows the user to specify up to 20 outer radii by which both the transmission and reflection surfaces will be divided into concentric, contiguous rings. The total electron and photon leakage (and the electron and photon energy spectra if requested) will be tallied in bins corresponding to these rings. The user interface is as follows:

(1) The simplest way to invoke the new tally is to include the card

\section{RINGS}

in the CYLTRAN input file. In this case, the transmission and reflection surfaces $w 11 l$ be subdivided into 10 concentric rings of equal surface area. 
(2) To obtain a different number of equal-area rings, the user simply adds an integer after the keyword:

\section{RINGS $\mathrm{n}$,}

where $\mathrm{n}$ must be at least one and no more than 20 .

(3) To choose user-defined radii, one uses the form

\section{RINGS $\mathrm{n}$ USER}

$\begin{array}{llll}R_{1} & R_{2} \cdot \cdot R_{n}\end{array}$

where the radii $R_{i}$ are entered in free format, beginning on the next line after the keyword, and $\mathbf{n}$ has the same limits as in (2). In this last case, it is not required that $R_{n}$ be equal to the radius of the problem cylinder. Thus, the user may tally only a portion of the transmission and reflection surfaces, corresponding for example to the area of a detector.

Note that the radii are always the outer radii of the rings, so the first ring (with inner radius zero) is always a disk. Fig. 18 illustrates option (3) above with $\mathrm{n}=4$ and with the last radius not equal to the radius of the problem cylinder.

When the RINGS keyword is used, a table is produced showing transmitted and reflected electrons and photons within each ring and for the total over all rings. If the outermost radius is not equal to the radius of the problem cylinder, this total will not include those particles transmitied or reflected beyond the last $r i n g$. This is a different (and slightly more flexible) approach from that taken in an earlier version of this tally (Ref. 32 ). The totals over the entire problem cylinder are still available under the heading "NUMBER AND ENERGY ESCAPE FRACTIONS."

If an electron escape energy spectrum is also requested by use of the ELECTRON-ESCAPE keyword with NBINE, an additiunal table is produced giving the distribution of transmitted and reflecte's electrons in energy bins for each ring and for the total over all rings. Again the total does not include any electrons escaping beyond the last ring, if the rings do not cover the problem cylinder. The normalization in this table is simply "per source particle," so that the entries represent just the number of electrons in each bin, not the 


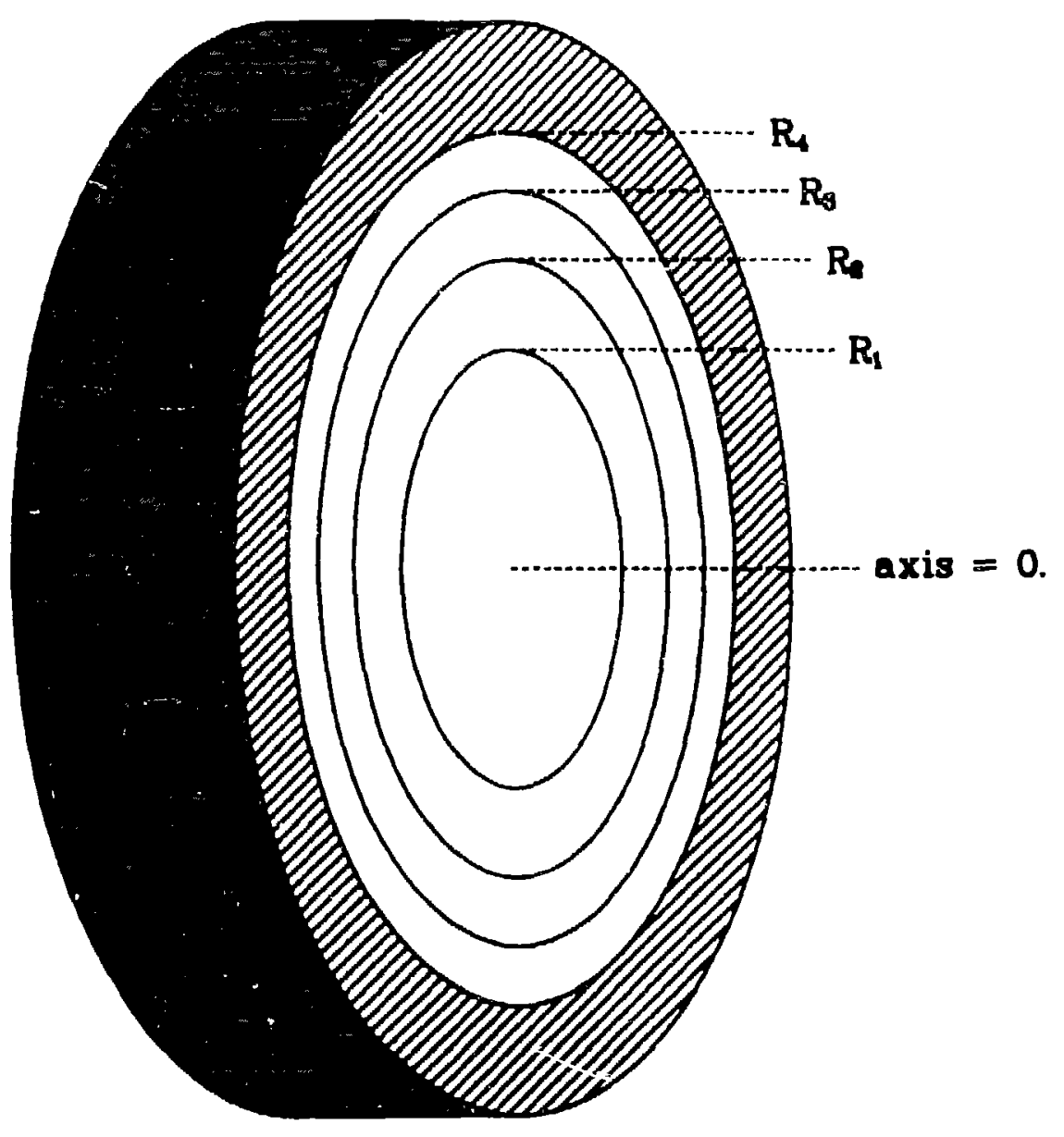

Fig. 18. Ring tally geometry superimposed on a transmission or reflection surface of the problem cylinder, illustrated for $n=4$. The ith ring is bounded by the radi $R_{1-1}$ and $R_{\text {. }}$. In this example, the rings are chosen to have equal surface areas. The cross-hatched region is the portion of the leakage surface outside the outermost tally ring. 
number per MeV. The totals over the entire problem cylinder are avallable under the heading "ENERGY SPECTRA OF ESCAPING ELECTRONS," where the normalization is "number per MeV."

Finally the keyword PHOTON-ESCAPE with NBINE together with RINGS will produce a table of transmitted and reflected photons in energy bing for each ring and for the total. The normalization will again be just "number in bin per source particle." The totals over the problem cylinder are given under "ENERGY SPECTRA OF ESCAPING PHOTONS," with the normalization "number per MeV."

The new tally is now included in the already-complled versions of the two codes/1ts/cyltran/cyltran and/1ts/cyltranp/cyltranp, and in the update files /its/cyltran/upcylt and/its/cyltranp/upcyltp. The tally should also work with user-supplied paiches for CYLTRANM, but this last application has not been tested. A more detailed description of a sample problem and the output associated with the ring tally is included in Ref. 33.

\section{CROSS SECTIONS AND PHYSICS}

A portion of our effort in Group $x-6$ is devoted to the acquisition, validation, and creation of libraries of cross sections for use in our deterministic and probabilistic codes. We also devote effort to supporting research, code development, and evaluation of atomic and nuclear physics models pertinent to our overall radiation transport effort.

A. Cross-Section Processing Codes on the CRAY (R. C. Littie)

Four codes are used all the time in the preparation and checking of continuous-energy neutron cr...s sections in ACE format for Monte C. $\%$ calculations.

- The code FISHP produces interpreted listings of ACE cross-section tables ready for microfiche output.

- The code MRKACR produces group-averaged cross sections from ACE format cross sections using a user-specified weight function in any userspecified group structure.

- The code TRLA is used to complete the process of translating data from the Livermore format ${ }^{34}$ into ACE format. The initial processing is carried out using MCPOINT; ${ }^{35}$ output files therefrom are polished using TRLA. 
- The code ADDXS is used to add reaction cross sections to an ACE crosssection table, the additional cross sections being available for use with the FM (tally multiplier) feature in MCNP. ADDXS is used mainly to incorporate the charged particle production cross sections into our ACE libraries.

These four codes are now up and running on CTSS machines in anticipation of making most of the ENDL-85 data library available to our Monte Carlo codes. The processing with MCPOINT can now be done only on the CRAY, so it was logical that the auxiliary processing also be done there. Detalls about source and executable files for these codes have been provided in Ref. 36.

B. Fission Product Cross Sections (R. E. Seamon and R. C. Little)

In calculations with fissionable materials, it is useful to have available cross sections for a "material" which represents the average neutronic properties of the resultant fission products. Over the years, these average cross sections have been called "fission product" cross sections. For years there have been sets of fission product cross sections available for use in Monte Carlo and discrete ordinates calculations. The earliest, available in multigroup form since about 1965, were based on work by Paul Whalen. The earliest continuous-energy Monte Carlo fission product cross sections available were the 1973 Livermore cross sections. 37 The ENDL fission product cross sections have changed through the years, and during this report period, the ENDL-85 fission product evaluation was made available for Monte Carlo calculations. 38

The whole matter of average neutronic properties for fission products was re-examined in the early $1980 \mathrm{~s}$, resulting in the Los Alamos evaluations for "prompt" fission products of U-235 and Pu-239. 39 These evaluations from ios Alamos Group $\mathrm{T}-2$ have been available in continuous-energy and multigroup forms since that work was completed.

Differences observed in certain neutron output calculations were at first attributed to differences between the Livermore and Los Alamos fission product cross-section evaluations. We were asked what would bring about such changes. Two memos were prepared 38,40 in which the cross sections, the angular distributions, and the secondary energy distributions were compared for not only the Livermore and the Los Alamos evaluations, but also for fission product 
'evaluations from Argonne National Laboratory ${ }^{41}$ and Westinghouse Hanford 42,43 where evaluations were produced for use in reactor calculations.

Calculational results indlcated more neutrons in the system when using Los Alamos nuclear rata. The Los Alamos $(n, 2 n)$ cross section has a threshold of about $4 \mathrm{MeV}$, whereas the Livermore $(n, 2 n)$ cross section has a threshold of about $8 \mathrm{MeV}$. Neutron multiplication because of the $(n, 2 n)$ reaction is obviously much greater when the Los Alamos fission product cross sections are used. The same situation exists for the $(n, 3 n)$ cross sections. Calculations using Livermore cross sections wlll have no contribution from the $(n, 3 n)$ reaction below $14 \mathrm{MeV}$, whereas the same calculations using Los Alamos cross sections will have a nontrivial $(n, 3 n)$ contribution. Above 1 keV, the Livermore capture cross section is roughly two orders of magnitude greater than the Los Alamos capture cross section. Therefore, calculations using Los Alamos fission product cross sections will have negligible neutron disappearance caused by absorption when compared to calculations using Livermore fission product cross sections. In summary, more neutrons from ( $n, x n)$ reactions and less neutron capture both contribute to the effects or served when the old ENDL73 or ENDL-85 Livermore cross sections are replaced by the Los Alamos cross sections.

This is by no means the end of the story. Many refinements in the controversial calculations have shown that the effects of changing just the fission product cross sections was not as large as initially thought. Furthermore, Howerton has also been looking critically at fission product cross-section calculations and has come up with a new fission product evaluation, a revised ENDL-85 version. We have seen plots of the cross sections therefrom; the $(n, 2 n)$ and $(n, 3 n)$ cross sections essentially match those of the Los Alamos evaluation. There remains a discrepancy in the ( $n$,gamma) cross section at low energies, the revised ENDL-85 graph $1 \mathrm{ying}$ still a decade higher than that for the Los Alamos evaluation. When the revised ENDL-85 evaluation is sent to Los Alamos, we shall make it available here in both continuous-energy and multigroup forms.

C. Multigroup Charged Particle Data (R. C. Little and F. E. Seamon)

We want to incorporate charged particle data into $\mathrm{X}$-Division multigroup codes--data for charged particles produced by many different incident particles such as neutrons, protons, and alpha particles. Little has proposed a format for a multigroup charged particle data base to include cross sections, reaction 
rates, and reaction-product spectra for any number of reactions involving any combination of incident particle and target 1sotope. Clearly, there is an enormous amount of data which can ultimately be incorporated into such a data base. The big challenge has been to incorporate into the format a fair amount of flexibility without allowing so much generality that the format becomes unusable.

Little has solicited comments on the format proposed in Ref. 44 because it is easier to change the format now than after many codes have "learned" the format. To help in gaining experience therewith, he prepared an initial version of a charged particle library containing elastic charged particle recoil matrices for five targets $(\mathrm{H}-1, \mathrm{D}-2, \mathrm{~T}-3, \mathrm{He}-3, \mathrm{He}-4)$. That CTSS library is stored at Los Alamos on CFS with universal read access under /MYREDSTUFF/CPDATA/CPFILE. The charged particle matrices themselves were prepared in Group T-2 by R. E. MacFarlane, as described in Ref. 45 . As MacFarlane has pointed sut, there are other reactions for those targets which produce charged particles; we have been glad to receive these elastic matrices to help us get started on using the proposed format.

Hand calculations of some of the elastic recoil matrix elements have been carried out using the kinematical formulae contained in Refs. 46 and 47 . We find a gratifying consistency between the expressions in Refs. 46 and 47 and the number calculated therewith, as transmitted in Ref. 45.

D. New Data (R. C. Little and R. E. Seamon)

1._-_Low-Energy Photon Cross Sections for MCNP. Low-energy photon interaction cross sections for hydrogen, helium, nitrogen, oxygen, and argon in the energy range $10 \mathrm{eV}$ to $1 \mathrm{keV}$ have been made available for use in MCNP. This work was done in response to a request from D. G. Collins, X-6, who needed good photon transport cross sections for this energy region. It is true that photons may be transported in MCNP at energies below $1 \mathrm{keV}$, but the cross sections used below $1 \mathrm{keV}$ will be the 1 -keV values avallable on the standard MCNP photon library MCPLIB.

The cross sections were extracted from Ref. 48 and merged with the MCPLIB values using a new code called ADDGXS. In the calculation of the heating numbers below $1 \mathrm{keV}$, it is assumed that the photon loses all of its energy; the heating numbers are therefore identical to the incident photon energies. 
Detalls concerning these speclal photon interaction cross sections are given in Ref. 49. In Ref. 49 are six figures which show that the total and partial cross sections join smoothly to the MCPLIB values at $1 \mathrm{keV}$.

2. Sc-45 Cross Sections for MCNP. At the request of C. D. Bowman, P-3, continuous-energy cross sections in ACE format have been made avallable for the single 1sotope of scandium, Sc-45. A complete cross-section evaluation for Sc45 did not exist; there were some partial dosimetry cross sections available, but there was no complete evaluation with dis easential reactions as well as energy and angular distributions. A complete evaluation is essential if one intends to transport neutrons through the material. Through the heroic efforts of P. G. Young, T-2, an evaluation for Sc-45 was prepared in ENDF/B format covering the energy range $1.0 \mathrm{e}-05 \mathrm{eV}$ to $5 \mathrm{MeV}$. The evaluation is described by Young in Ref. 50 .

The new evaluation was processed here in Group $X-6$ by Little through the RECONR, BROADR, HEATR, and ACER modules of NJOY to produce an ACE format crosssection file at $300 \mathrm{~K}$ for use by Bowman. It is understood that the data are valid only to $5 \mathrm{MeV}$; the data were extended to $20 \mathrm{MeV}$ for completeness only, to make the energy range consistent with other ACE cross-section files. The detalls of that work and the location of the output files are given in Ref. 51.

3. Y-89 Cross sections for MCNP. Two sets of continuous-energy cross sections for $Y-89$ have been made available for use with MCNP: one is from ENDF/B-V, 52 the other from the 1985 version of the Livermore library (ENDL85). 35 The ENDF/B-V evaluation is found as MAT $=9202$ on Tape 542, the so-called Fission Product Tape. Although this evaluation contalns enough information to qualify it as "complete" in that it can be used for the transport of neutrons, it is by no means as detailed as the evaluations found on the general purpose ENDF/B-V files. The Y-89 evaluation found on the April 24,1985 , version of ENDL-85 was processed using MCPOINT. Little's new postprocessing code ENDLGAM was then used to incorporate the photon production data in expanded ACE format. 53 There is a surprising amount of detall provided in the ENDL-85 evaluation.

The preparation of these ACE files is detailed in Ref. 54. As mentioned there, these two evaluations are very different and their characteristics should be well understood before using them. Details given in Ref. 54 , including a disagreement of a factor of 2 in the total cross section below 
$1 \mathrm{keV}$, caused the ENDF/B-V evaluator (R. E. Schenter, HEDL) to re-examine his work and to suggest a modification of his evaluation for Y-89. Following Schenter's suggestion, the scattering radius was changed and the modifled evaluation was reprocessed through the RECONR, BROADR, HEATR, ad ACER modules of NJOY. Although there are still remarkable differences between the modified ENDF/B-V and ENDL-85 Y-89 cross sections, the total cross section from these two sources is now in good agreement. Schenter had indicated that a complete re-evaluation of $Y-89$ is likely for ENDF/B-VI.

The ENDF/B-V Y-89 ACE format cross sections have been replaced with the modified results. This work is detailed in Ref 55.

4. Cross Sections for Eu, Gd, and Ho- 165 from ENDL-85 in Cont inuousEnergy and Multigroup Form. There is a need for Eu, Gd, and Ho-165 cross sections with photon production data in both continuous-energy and multigroup format.

- We have had isotopic evaluations from ENDF/B-V for europium available; there is photon production data for two of the four isotopes, Eu-151 and Eu-153. There were also data for natural europium from ENDL-73 and ENDL-79 without photon production data. The ENDF/B-V evaluations were available in both pointwise and multigroup form; the others were available in pointwise form only.

- We have had isotopic evaluations of gadolinium available without photon production from ENDF/B-V. There have also been natural gadolinium data from ENDL-73 and ENDL-79 without photon production. These gadolinium cross sections were available in pointwise form only.

- We have had ENDL-73 and ENDL-79 cross sections for Ho-165 available in pointwise form only.

From the three items above, clearly it has been Impossible to make discrete ordinates and corresponding Monte Carlo calculations using these materials. The April 24, 1985, version of ENDL-85 includes data with photon production for natural europium, natural gadolinium, and Ho-165. We have processed these materials into both expanded ACE format and multigroup format and made them available for general use at Los Alamos.

The processing of the three evaluations into expanded ACE format is described in Ref. 56. These are the first ACE format data tables derived from 
Livermore evaluations that allow the neutron-induced photon spectra to be sampled in MCNP as the evaluator intended. All previous Livermore data sets for $\mathrm{Eu}, \mathrm{Gd}$, and Ho-165 should be considered obsolete.

The processing of the three evaluations into multigroup format is described in Ref. 57 ; the avallability on our most complete multigroup libraries, MENDF5X and MENDF5GX, is discussed in Ref. 58. There are now companion continuous-energy and multigroup libraries, including photon production, available for these three materials. We continue to feel that data from the ENDF/B-V evaluations of Eu-151 and Eu-153 are the best coupled data sets available. The natural europium data sets from ENDL-85 were prepared for completenes a and to allow intercomparison with other codes at Los Alamos which use only ENDL data.

E. Physics Sources of the ITS Coupled Electron/Photon Monte Carlo Transport Codes (Particularly CYLTRAN) (J. J. Devaney)

The physics sources of the Integrated Tiger Series (ITS) Codes ${ }^{29}$ have been reviewed. CYLTRAN ${ }^{59}$ is a simpler physics ITS member whose geometry is cylindrically symmetric, but has full three-dimensional particle trajectories. The IIS codes (1984) were developed and are maintained by Sandia National Laboratory. They are largely based on the earlier Sandia code SANDYL (1973) 60 and the National Bureau of Standards codes DATAPAC $4^{61}$ (1968) ard ETRAN $15^{62}$ (1968).

These codes are highly sophisticated. They (ITS) calculate the trarisport photons and their mutual interactive production in the energy range trom $1 \mathrm{GeV}$ to $1.0 \mathrm{keV}$. They use electron stopping powers, electron elastic scattering, electron impact ionization, Auger electron production, fluorescence, (in Pcodes: of the K, LI, LII, LIII, $\langle M\rangle$, and $\langle N\rangle$ shells, $\rangle$ is average), bremsstrahlung, photoelectric effect, Compton effect, pair and triplet production, annihilation, Raleigh scattering, etc. In consequence, although our study took longer than expected, it is restricted to the sources of the physics. The actual codes and the particular form of the physical formulas used were not reviewed.

In brief, the authors of these codes appear to use the best physics avallable that have practical suitability. The treatment is sophisticated. The ITS system is the culmination of more than 15 years of effort by many different people. The codes have been tested against experiment in several contexts. The earlier codes have differences relative to experiment from - 0 
to - $40 \%$ (to a factor 3 in one case) for particular physical configurations. A CYLTRAN calculation of thick target bremsstrahlung yields gives differences relative to experiment of from 0 to - 20\%. A SANDYL electron back scattering calculation achieved - 10\% relative to experiment. Another SANDYL calculation had accuracies of $-20 \%$.

The codes are continually being updated as new studies become avallable and as the various authors find time to do so. Common to them, as to many of us, are divided responsibilities that preclude sole attention to electron/ photon transport. The code authors appear to recognize the remaining principal areas needing improvement. The analyses, therefore, are offered not as criticism but rather to acquaint the reader with the type of physics used and with its accuracy, as best we can determine it.

My overall impression of the accuracy of the physics is that it is typically 10-20\%. But some physics is better and some worse. As is wall known, physics errors do not always translate into calculation result errors in a one-to-one fashion. For instance, a large error, say in an angular distribution at only one extreme, will have negligible effest (that is, little error) if the whole distribution is important to the result, but that error will have a dominant effect (large error) if that particular extreme dominates calculation of the result.

A well-racognized poor approximation for bremsstrahlung cross sections for high-z (and low-energy electrons) is the use of the Born Approximation (Bethe-Heitler), which can give rise to errors of over a factor of 10; however, empirical corrections lead to improvements in the ITS codes, so the practical result-for example, for thick target bremsstrahlung spectra--is an expected error of only 20 to $30 \%$. Impraved (<10\% error) cross sectiuns are in the works.

Stopping powers have errors of 0 to $25 \%$ to $300 \%$, decreasing to $\leq 20 \%$ at electron energies $210 \mathrm{keV}$. An improved stopping power data algorithm has been developed. Electron scaitering accuracies range from 10\% to $20 \%$. $x$-ray cross sections have accuracies ranging from $1 \%$ to $5 \%$ to $>10 \%$. K fluorescent yields are good to $0.5 \%$. Electron impact ionization is from as good as experimental error to - $30 \%$ or - $40 \%$ error. Better K-shell ionization results exist but are not yet in the codes.

In summary, the ITS series of codes have good physics, capable of further improvement at the cost of further analysis and experiment. The codes have 
considerable physlcal detail. The codes do not treat mutual electromagnetic interactions of charges and so are not intended to be used to calculate high charge and current densities.

\section{REFERENCES}

1. R. Douglas O'Dell, Forrest W. Brinkley, Jr., and Iuane R. Marr, "User's Manual for ONEDANT: A Code Package for One-dimensional, DiffuionAccelerated, Neutral-Particle Transport," Los Alamos National Laborjtory

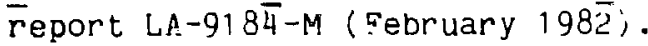

2. Ray E. Alcouffe, Fornest W. Brlrtley, nuane R. Marr, and R. Douglas C'Dell, "User's Guide for TuJDANT: A Code Package for TwoDimensional, Diffusion-Accelerated, Neutral-Particle Transport," Los Alamos National Laboratory report LA-10049-M Rev. 1 (Cctober 1984).

3. R. D. O'Dell, "Radiation Transport, January 1, 1985-March, 31, 1985," Los Alamos National Laboratory report LA-10591-PR (January 1986).

4. R. D. O'Dell, "Standard Interface Flles and Procedures for Reactor Physics Codes, Veryion IV, "Los Alamos Scientific iaboratory report LA-6941-MS (September: 1977).

5. Wallace E. Walters, Forrest W. Brinkley, and Duane R. Marr, "User's Guide for TWOHEX. A Code Package for Two-Dimensional, Neutral-Particle Transport in Equilateral Triangular Meshes," Los Alamos National Laboratory report LA-i0258-M (October 1984).

6. R. E. Alcouffe, "Diffusion Synthetic Acceleration Methods for the Diamond-Differenced Discrete-Ordinates Equations," Nucl. Sci. Eng., 64, $344-355(1977)$.

i. R. D. O'De11, "Radiation Transport. April 1, 1983-December 31, 1983," Los Hlamos National Laboratory report LA-10189-PR (October 1984).

8. E. W. Larsen, "Diffusion Theory as an Asymptotic ilmit of Transport Theory for Nearly Critical Systems with Small Mean Free Paths,".$n n$. Nucl. Energy. I, $249(1980)$.

9. E. E. Lewis and W. F. Milier, Jr., Computational Methods of Neutron Transport (Wiley, New York, 1984).

10. C. M. Lund and J. R. Wilson, "Some Numerical Methods for Time-Dependent Multifrequency Radiation Transport Calculations in One Dimension," Lawrence Livermore National Laboratory report UCRL-84678 (1300).

11. J. I. Castor, "Comparison of the Errors of the Wilson and Feautrier Schemes for Differencing the Equation of Transfer, as Applied to a Class of Simple Model Problems," Lawrence Livermore National Laboratory memorandum (Jan. 29, 1982) and addendum (Feb. 2, 1982).

12. E. W. Larsen, J. E. Morel, and W. F. Miller, Jr., "Asymptotic Solutions of Numerical Transport Problems in Diffusive Regimes, "Lcs Alamos National Laboratory document LA-UR-86-69 (1986). 
13. G. C. Fomraring, The Equations CS R diation Hydrodynamics (Pergamon Press, Oxford, 1973).

14. B. Mercier, "Applications of Accret:ve Operat:" Theory to the Radiative Transfer Equations," SIAM J. Math. Anal., to be published.

15. F. Golse and B. Perthame, "Generalized Solutions of the Radiative Transfer Equations in a Singular Case," CEA-N-2451 (1985).

16. J. A. Fleck, Jr, and J. D. Cummings, "An Implicit Monte Carlo Scheme for Salculating Time and Frequency-Dependent Nonlinear Radiation Transport," ․ Comp. Phys. $8,313(1971)$.

17. Raymond E. Alcouffe, Brailey A. Clark, and Edward W. Larsen, "The Diffusion Synthetic Acseleration of Transport Iterations, with Application to a Radiation Hydrodynamics Problem," in Computational Techniques, Multiple Time Scales, J. U. Brackbill and B. I. Cohen, Eds. (Academic Press, New York, 1985).

18. J. E. Morel, E. W. Larsen, and M. K. Matzen, "A Synthetic Acceleration Scheme for Radiative Diffusion Calculations," J. Quant. Spectrose. Radiat. Transfer, to be published.

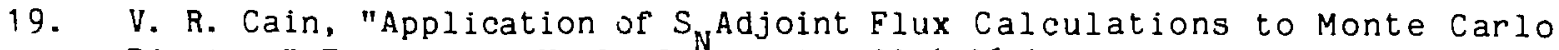
Biasing," Trans. Am. Nucl. Soc., 10, 399 (1967).

20. R. E. Alcouffe, "A First Collision Source Method for Coupling Monte Carlo and Discrate Ordinates for Localized Source Problems," to appear in Springer Verlag, Lecture Notes in Physics, No. 240, 1985.

21. W. L. Filippone and B. D. Garapol, "An $\mathrm{S}_{\mathrm{N}}$-Monte Carlo Hybrid Method," Trans. Am. Nucl. Soc. $\underline{41}, 487$ (1982).

22. W. L. Filippone, S. Woolf, and R. Layigne, "Particle Transport Calculations with the Method of Streaming Rays," liucl. Sci. Eng., 77, 119 (1980).

23. W. L. Filippone and S. Woolf, "Application of the Method of Streaining Rays to Particle Transport in Complex Geometries," invited paper to the ANS/ENS International Topical Meeting on Advances in Mathematical Models Ior Nuclear Engineering Problems, Munich, Federal Republic of Germany, Vol. I, 67, (1981).

24. B. A. Clark, "The Streaming Matrix Hybrid Methou for Discrete-Ordinates Calculations," Proc. ANS Topical Meeting, Advances in Reactor Computations, March 28-31, 1983, Salt Lake City, 1, 135 (1983).

25. W. L. Filippone, "Orders-of-Scattering Expansion Functions," Trans. Am. Nucl. Soc., 40, 649 (1980).

26. Los Alamos Monte Carlo iroup, "MCNP - A General Monte Carlo Code for Neutron and Photon Transport, Version 2B," Los Alamos National Laboratory report LA-7396-M, Rev. (April 1981). 
27. R. C. Little, "Proposa: to Eliminate Four Sampling Schemes from MCNP," Los Alamos National Laboratory memorandum X-6:RCL-85-305 (June 13, 1985).

28. "HETC - Monte Carlo High-Energy Nucleon-Meson Transport Code," Radiation Sinielding Informat on Center report CCC-178, Oak Ridge National Laboratory (1977).

29. J. A. Halbleib and T. A. Mehlhorn, "ITS: The Integrated TIGER Series of Coupled Electron/Photon Monte Carlo Transport Codes," Sandia National Laboratories report SAND 84-0573 (Albuquerque, New ilexico, November 1984 j.

30. T. S. Subramamian, J. L. Romeru, F. P. Brady, J. W. Watson, D. H. Fitzgerald, R. Garrett, G. A. Needham, J. L. Ullmann, and C. I. Zanelli, "Double Differential Inclusive Hydrogen and Helium Spectra from NeutronInduced Reactions on Carbon at 27.4, 39.7, and 60.7 MeV," Phys. Rev. C, 28 (1983), p. 521 .

31. H. Grady Hughes and Joseph M. Mack, "Integrated TIGER Ser les at Los Alamos," Los Aiamos National Laboratory memorandum X-6:HCH-85-3C6 (June 17, 1985).

32. J. M. Mack and H. G. Hughes, "Radial Leakage Tally and Generalized Conical Source for CYLTRAN," Los Alamos National Laboratory memorandum $\mathrm{X}-6: 83-7-8$ (July 8,1983 ).

33. H. Grady Hughes, "Ring Tally for CYLTRAN," Los Alamos National Laboratory memor andum $\mathrm{X}-6: \mathrm{HGH}-85-427$ (August 23,1985 ).

34. R. J. Howerton, R. J. Doyas, T. C. Michels, and S. T. Perkins, "An Integrated System for Production of Neutronics and Photonics Calculational Constants, Volume 4, Evaluated Nuclear Cross-Section Library," Lawrence Radiation Laboratory report UCRL-50400, (April 15, 1971).

35. R. J. Howerton, R. E. Dye, P. C. Giles, J. R. Kimlinger, S. T. Perkins, and E. F. Plechaty, "OMEGA: A CRAY 1 Executive Code for LLNL Nuclear Data Libraries," Lawrence Livermore National Laboratory report UCRL-50400 Vol. 25 (August 1983).

36. R. C. Little, "Cross-Section Processing Codes on the CRAY," Los Alamos National Labcratory memorandum $X-6: \mathrm{RCL}-85-415$ (August 13, 1985).

37. R. J. Howerton and M. H. MacGregor, "The LLL Evaluated Nuclear Data Library (ENDL): Descriptions of Individual Evaluations for Z=0-98," Lawrence Livermore National Laboratory report UCRL -50400 Vol. 15, Rev. 1 (May 17, 1978).

38. R. E. Seamon and R. .. Little, "Fission Product Cross Sections from ENDL85," Los Alamos National Laboratory memorandum X-6:RES:RCL-85-372 (July 19, 1985).

39. D. G. Foster, Jr. and E. D. Arthur, "Average Neutronic Properties of "Prompt" Fission Products," Los Alamos National Laboratory report LA-9168-MS (February 1932). 
40. R. C. Little and R. E. Seamon, "Comparing Fission Product Cross Sections," Los Alamos National Laboratory memorandum $X-6: R C I$ :RES-85-404 (August 7, 1985).

41. J. R. Liaw and H. Henryson II, "Lumped Fission Product Neutron Cross Sections Based on ENDF/B-V for Fast Reactor Analysis," Nucl. Sci. Eng. 84, , 324 (1983).

42. R. E. Schenter, "Fission Product and Actinide Subcommittee Evaluation Report," Appendix 3C of "Summary of the CSEWG Meeting, May 13-15, 1981," edited by $S$. Pearlstein (July 1, 1981).

43. J. R. White and R. E. Schenter, "Analysis of the Lumped Fission Product in CRBR," Trans. Am. Nucl. Soc. 39, 931 (1984).

44. R. C. Little, "X-Division Charged-Particle Library Format (1)," Los Alamos National Laboratory memorandum X-6:RCL-85-459 (September 17 , 1985).

45. R. E. MacFarlane, "30-Group Elastic Recoil Matrices," Los Alamos National Laboratory memorandum $\mathrm{T}-2-\mathrm{M}-i 621$ (July 10, 1985).

46. L. L. Carter and E. D. Cashwell, "Particle-Transport Simulation with the Monte Carlo Method," ERDA Critical Review Series TID-26607 (1975).

47. R. E. MacFarlane, "ENDF'B Format Change," Los Alamos Scientific Laboratory memorandum T-2-L-4021 (April 21, 1981).

48. R. B. Livesay, "Photon Cross Sections for $10 \mathrm{eV}<E<100$ MeV," Radiation Research Associates report RRA-T750ó (June 30, 1975).

49. R. C. Little, "Low-Energy Photon Cross Sections (10 eV - 1 keV) for MCNP," Los Alamos Natical Laboratory memorandum X-6:RCL-85-428 (August 26, 1985).

50. P. G. Young, "Cross Section for $n+S c-45$," Los Alamos National Laboratory memorandum T-2-M-1641 (August 28, 1985).

51. R. C. isittle, "Sc-45 Cross Sections for MCNP," Los Alamos National Laboratcry memorandum X-6:RCL-85-430 (August 27, 1985).

52. R. Kinsey, ed., "ENDF-102, Data Formats and Procedures for the Evaluated Nuclear Data File, ENDF/B-V," Brookhaven National Laboratory report BNL-NCS-50496 (ENDF-102) (November 1983).

53. R. C. Li"tle and R. E. Seamon, "Neutron-Induced Photon Production in MCNP," Proc. of the 6th Intl. Conf. on Radiation Shielding, Vol. I, 151 (May 1985).

54. R. C. Little, "Y-89 Cross Sections ror MCNP," Los Alamos National Laborato.y memorandum X-6:RCL-85-419 (August 16, 1985).

55. R. C. Little, "Modified ENDF/B-V Y-89 Cross Sections for MCNP," Los A lamos National Laboratory memorancium X-6:RCL-85-443 (September 6, 1985). 